\title{
Design and Evaluation of an Observer-Based Disturbance Rejection Controller for Electric Power Steering Systems
}

Proceedings of the Institution of Mechanical Engineers, Part D: Journal of Automobile Engineering 000(00):1-13

(C) The Author(s) 2010 Reprints and permission: sagepub.co.uk/journalsPermissions.nav DOI:doi number http://mms.sagepub.com

\author{
Naser Mehrabi*, John McPhee and Nasser L. Azad \\ Systems Design Engineering, University of Waterloo, Canada
}

\begin{abstract}
The goal of this paper is to develop an observer-based disturbance rejection Electric Power Steering (EPS) controller to provide steering assistance and improve the driver's steering feel. For the purpose of control design, a control-oriented model of a vehicle with a column-assist EPS system is developed and verified against a high-fidelity multibody dynamics model of the vehicle. The high-fidelity model is used to mimic vehicle dynamics to study controller performance in realistic driving conditions. Then, a Linear Quadratic Gaussian approach is used to design an EPS optimal controller, in which a Kalman filter estimates the unmeasured steering system's states and external disturbance. A new formulation for the Linear Quadratic Regulator objective function is proposed to take advantages of the known information about the system dynamics to attenuate the disturbance and magnify the driver's torque.

Finally, the EPS controller is applied to the high-fidelity vehicle model in a software-in-the-loop simulation to evaluate its robustness and performance under realistic conditions. The results show that the proposed controller can effectively reduce the disturbance induced in the steering rack, and simultaneously magnify the driver's steering torque by use of a bi-linear EPS characteristic curve. Then, to show the disturbance rejection properties of this EPS controller, its performance is compared to $\mathrm{H}_{2} / H_{\infty}$ and PID control designs using time and frequency domain analysis.
\end{abstract}

Keywords

electric power steering; optimal control; LQG; observer-based disturbance rejection; noise

\section{Introduction}

Many automobile manufacturers are switching to Electric Power Steering (EPS) systems for better performance and costeffective advantages of EPS systems over the traditional Hydraulic Power Steering (HPS) systems. Compared to HPS, EPS offers lower energy consumption, lower total weight and higher package flexibility with no extra cost. In addition, under increasing fuel prices, the fuel saving property of EPS makes these products more economical than HPS. Furthermore, an EPS actuator can manipulate the assist torque independent of engine or vehicle operating condition; therefore new control strategies can be developed to simultaneously improve the driver's steering feel and safety of the vehicle.

\footnotetext{
*Corresponding author; e-mail: nmehrabi@uwaterloo.ca
} 


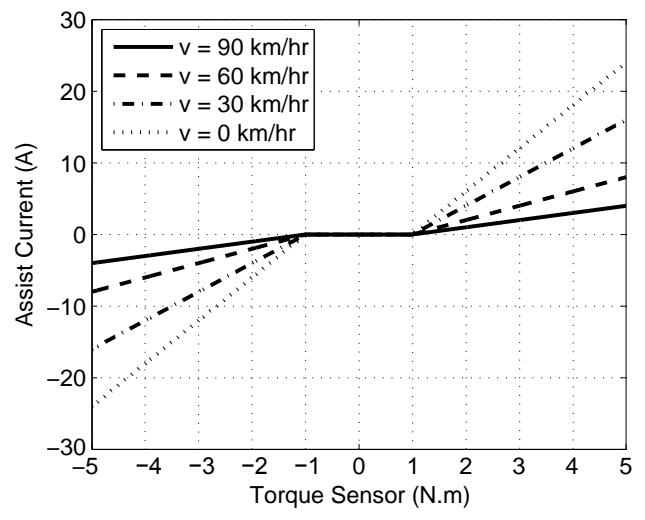

(a)

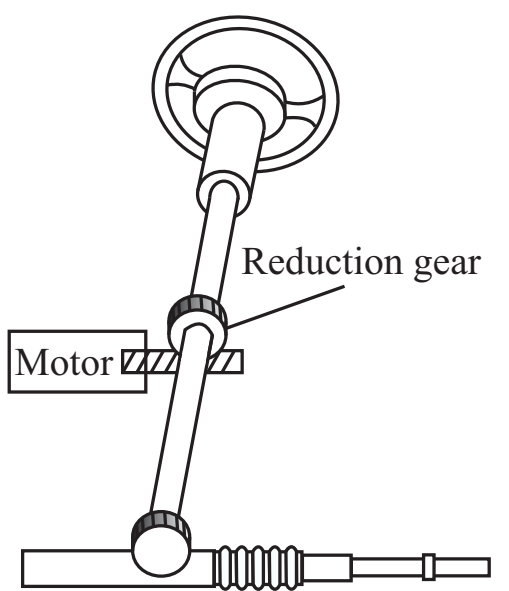

(b)

Fig. 1. (a) An example of bi-linear characteristic curves of Electric Power Steering systems (b) Column-assist Electric Power Steering (EPS) system

The main responsibility of EPS systems is to reduce the physical effort of the driver. Therefore, the EPS assist torque should compensate most of the resistive torque at the steering wheel. As a result, almost all power steering systems have an assistance component in their logic to produce an assist torque as a function of the driver torque. This relation is typically presented in so-called characteristic curves (also called boost curves or torque maps) [1]. The shape and dynamics of these curves were studied in the literature to provide better assistance and steering feel for drivers $[2 ; 3 ; 4 ; 5 ; 6]$. Typically, the steering characteristic curves are bi-linear or multi-linear functions of the steering torque. For example, Fig. 1a shows a typical steering characteristic curve which is a bi-linear function of the column torque, where the rate of assistance varies with the vehicle speed. This characteristic curve consists of a no-assist zone to avoid the off-center feeling, and a linear steering assistance zone. A similar characteristic curve is utilized in the EPS controller that we propose.

The steering system interacts with three unknown external torques: driver steering torque, self-aligning torque and external torques due to environmental excitations. EPS systems usually use a torque sensor to approximate the driver steering torque, but there is no sensor to measure the other torques. Hence, designing an EPS controller is a very challenging problem, since it requires solving a tracking problem under the existence of unknown disturbance and uncertainty. A wide range of control strategies have been reported in the literature to be used to design EPS controllers, including classical, model-based, and black-box control methods. The robust control approach has been regularly used in the EPS control design to improve robustness and performance of the system. $H_{\infty}$ synthesis is used in $[7 ; 8 ; 9 ; 10]$ to provide assistance, while simultaneously minimizing the effect of disturbances on the outputs. The Linear Quadratic Gaussian (LQG) control method also has been used in designing EPS controllers. LQG control is used to reduce the number of required sensors and to provide assistance in presence of external disturbance and measurement noise $[11 ; 12 ; 13]$. It is likely to say that the current state-of-the-art in EPS control design is the $H_{2} / H_{\infty}$ approach, since it gathers both $H_{2}$ and $H_{\infty}$ advantages together. Zhao et al. [14] designed a mixed $H_{2} / H_{\infty}$ controller to obtain better system performance by incorporating $H_{2}$ and $H_{\infty}$ norms of performance indices in the controller optimization. Similar approach has been used in [15; 16], where the passivity constraint of the human drivers is considered in the design to ensure the closed-loop stability.

Relatively less effort has been made in designing friction compensation and disturbance observer controllers for EPS systems. A friction compensation control is developed in [17] by considering an equivalent friction model of the worm gear. Then, the prediction of this model is used to compensate for the friction through motor torque. Dannöhl et al. [18] developed a modified $H_{\infty}$ controller for a rack-assisted EPS by considering separate unknown external forces to model friction at the steering wheel, rack and motor. The modified controller enhanced the performance of the system in comparison with 
the original $H_{\infty}$ controller. To suppress periodic disturbances (shimmy) at the steering column, disturbance compensation controllers have been developed. In these controllers, the periodic disturbance is extracted from the measured steering torque using an analytical approach [19] or by use of a non-linear low-pass filter [20], and then compensated through the actuator. A disturbance observer also has been developed to estimate the resistive steering torque and driver steering torque using an extended state observer [21], or Kalman filter [22] or sliding mode observer [23]; this estimation is then used to produce a desired assistance in the presence of external disturbances.

In this research, an observer-based disturbance rejection EPS controller consisting of a Kalman filter and a Linear Quadratic Regulator (LQR) is developed based on a control-oriented model of a vehicle. Although this control-oriented model is linear, nonlinear effects such as external disturbances and friction have been considered in the control design. The Kalman filter observer estimates the control-oriented model states and the applied disturbances to the steering system such as those induced by the interaction between the vehicle and the road, or by the friction forces acting between the steering components. To improve the disturbance estimation, the steering wheel angle measurement is assumed to be a known input to the Kalman filter, and the remaining measurements are assumed to be measured outputs. This simple rearrangement removes the uncertainty due to the unknown driver's torque in the Kalman filter. The estimated disturbance and states are augmented with the measured steering wheel angle and sent to the EPS controller. This new control configuration provides feedback of all states and estimation of the disturbance to a disturbance rejection LQR. A new formulation for the LQR objective function is introduced, in which the EPS generates an opposing torque to cancel the disturbance while magnifying the driver's torque based on a bi-linear characteristic curve. The proposed controller shows robustness against measurement noise and external disturbances, and improves the EPS performance in comparison with common EPS controllers.

In this paper, first a high-fidelity vehicle model including a column-assisted EPS system is developed. Then, for the purpose of controller design, a control-oriented model of the vehicle and EPS system is developed and verified against the high-fidelity model. In the third section, an observer-based optimal disturbance rejection controller is designed and applied to the high-fidelity model. Simulation results and controller validation are presented in the fourth section. Finally, the paper is summarized and conclusions are made.

\section{Dynamical Modeling}

A clear understanding of the dynamics of a system is crucial in designing control systems, since not only does it strengthen our knowledge of the system but also it reduces development time and cost. Therefore, in this article, a full vehicle model including a column-assisted EPS system is developed in MapleSim ${ }^{1}$ using a multibody dynamics approach. This model is used to study and evaluate an EPS controller. Although the high-fidelity model is suitable to study the system's dynamics, it is not suitable to be used within a model-based controller due to its non-linearities and complexities. A low-order control-oriented model that preserves the important dynamics of the system is required for the controller.

In this section, a high-fidelity multibody dynamical model of vehicle and a control-oriented model are presented, and the accuracy of the control-oriented model is verified against the high-fidelity model.

\subsection{Full Vehicle Model in MapleSim}

In this research, a vehicle model with a front MacPherson suspension and a rear multi-link/semi-trailing arm suspension typical in an SUV (Sport Utility Vehicle) is developed as shown in Fig. 2a. The developed vehicle model consists of 50 generalized coordinates coupled by 34 algebraic equations. This model in total has 16 degrees of freedom (DOF). Six DOF are associated with the rigid body motion of the chassis, four are related to the wheels' spin, four are related to the suspensions' vertical motion and two are associated with the steering motion.

\footnotetext{
${ }^{1}$ MapleSim is a registered trademark of Maplesoft
} 


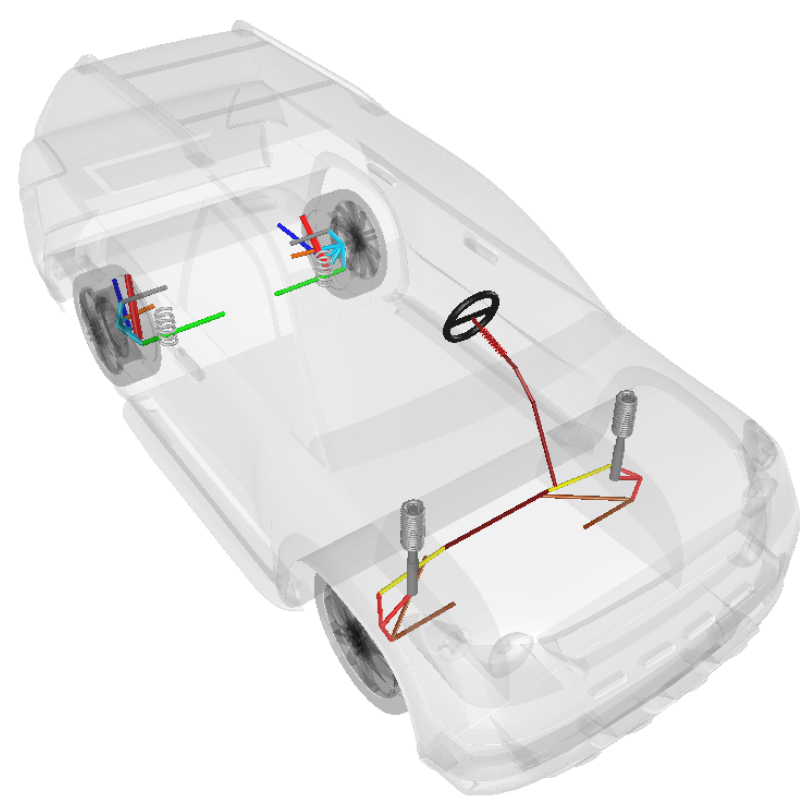

(a)

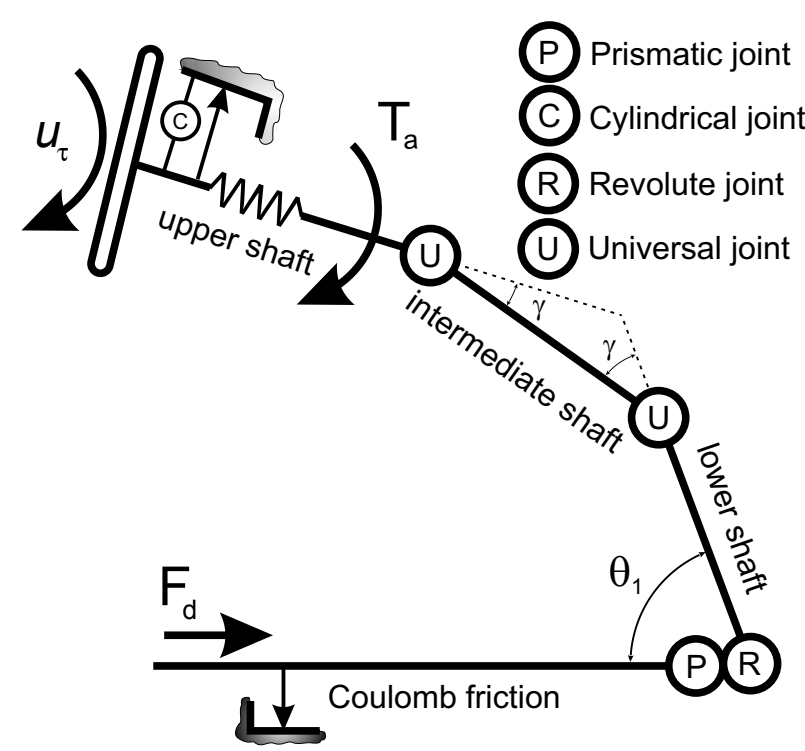

(b)

Fig. 2. (a) A view of the vehicle model in MapleSim, (b) schematic view of steering system in MapleSim, including the location of driver torque $\left(u_{\tau}\right)$, assist torque $\left(T_{a}\right)$, disturbance torque $\left(u_{d}=F_{d} r_{p}\right)$, and Coulomb friction

Chassis and Suspension. A conventional MacPherson suspension consists of a lower control arm, spindle and strut. The lower control arm connects the chassis to the spindle allowing vertical movements of the tire. The strut, including a co-linear spring and shock absorber, is mounted on the spindle to carry the vehicle weight and reduce the vibrations passed to the body. Similarly, in the multi-link/semi-trailing arm rear suspension, the suspension arms connect the chassis to the spindle allowing only vertical tire movements. A spring and a shock absorber are mounted separately on the arms of the rear suspension to isolate the body from the road irregularities. A similar mechanism to that shown in [24] has been used to construct the multi-link rear suspension. The kinematics of the suspension systems are verified against an experimentally validated ADAMS model. More information about the high-fidelity vehicle model can be found in [25;26].

Steering System Model. Since the steering system is the focus of this research, the model construction is described in detail. A conventional rack and pinion steering system consists of two universal joints that connect the three steering column shafts to transmit the rotational motion of the steering wheel to the pinion. The steering wheel is connected to the steering column housing by a cylindrical joint allowing rotational and telescopic movements of steering column, and at the other end, a rack-and-pinion gear is used to transform the rotational motion of the lower steering shaft to the translational motion of the rack with a specified reduction ratio. As shown in Fig. 2b, the rack and pinion steering system is a one-degree of freedom mechanism, in which rotating the steering wheel results in a unique displacement of the rack and the wheel.

Torsion bars (torque sensors) are an essential part of electric and hydraulic power steering systems and the major source of flexibility in the steering systems. In the high-fidelity model, a torsion bar is placed in the upper steering shaft by using a torsional spring and damper representing the shaft elasticity properties. By registering the relative angular displacement between the two ends of the bar, and by assuming the elastic behavior of shaft, the torque transferring through the shaft can be approximated as follows:

$$
T_{t b}=K_{t b} \Delta \theta_{t b}+C_{t b} \Delta \dot{\theta}_{t b}
$$

where $K_{t b}$ and $C_{t b}$ are the stiffness and damping coefficients of the torsion bar, and $\Delta \theta_{t b}$ is the torsion bar deflection. 


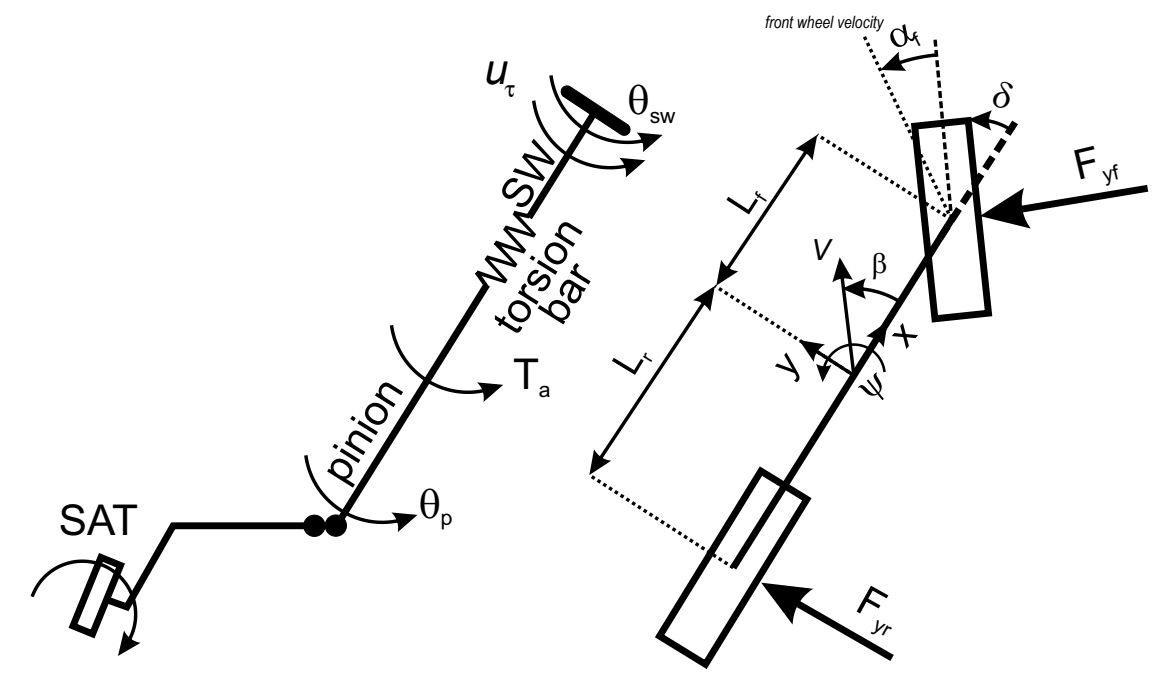

Fig. 3. Schematic view of the control-oriented model confined in the LQG controller including a linear bicycle model (right) with a column-assisted EPS system (left)

Direct current (DC) electric motors connecting through a worm gear to the steering column are commonly used in EPS systems to provide the assist torque [27]. In this article, a first-order differential equation is used to simulate the DC electric motor dynamics. This model is given by:

$$
\begin{gathered}
L_{m} \frac{\mathrm{d} i}{\mathrm{~d} t}+R_{m} i+K_{e} \dot{\theta}_{m}=u_{v} \\
T_{e}=K_{e} i
\end{gathered}
$$

where $i$ and $u_{v}$ are the current and terminal voltage of the DC motor, $L_{m}, R_{m}$, and $K_{e}$ are the inductance, resistance and back electromotive force (emf) coefficients of the electric motor, and $T_{e}$ and $\dot{\theta}_{m}$ are the motor torque and angular velocity of the motor shaft.

In the physical system, friction is generated at the mechanical connections between the steering wheel and its housing, the rack and its housing and in the worm gear [28]. In this article, a friction model [29] representing the Coulomb and viscous part of the friction force as given in Eq. (3) has been used to simulate the steering wheel and rack frictions.

$$
F_{f r i c}(t)=b v_{r e l}^{a}+\tanh \left(\frac{v_{r e l}}{v_{0}}\right)\left(f_{c}+\left(f_{s}-f_{c}\right)^{-\left|\frac{v_{r e l}}{v_{s}}\right|^{n}}\right)
$$

where $b$ and $a$ are the viscous damping coefficient and exponent and $f_{s}$ and $f_{c}$ are the Coulomb friction coefficients. $v_{r e l}$ is the velocity of the steering column and rack relative to their housings and $v_{s}, v_{0}$ and $n$ are shaping factors. The numerical value of the parameters used in the steering system are shown in Table. 1.

\subsection{Control-Oriented Model of EPS System}

A simplified control-oriented model is developed for use in the EPS controller. Since the EPS controller has to perform in real-time, and since the amount of memory and process time allocated to steering control in the vehicle's Electronic Control Unit (ECU) are limited, a simplified control-oriented model is used to minimize the computational resources required.

As shown in Fig. 3, the control-oriented model includes the steering column $\left(u_{d}\right)$, electric motor $\left(T_{a}\right)$, and lateral dynamics of vehicle. These steering components have been selected because of their important influence on the steering dynamics. For example, the lateral dynamics of the vehicle have been included in this model to predict the lateral force of front wheels and the resistive steering (self-aligning) torque. The flexibility of the torsion bar appears in two differential 
equations (corresponding to steering wheel and pinion) to capture the dynamics of steering shaft. Considering the moment of inertia and the viscous damping of a steering wheel, the steering wheel equation of motion is obtained:

$$
\begin{gathered}
J_{s w} \ddot{\theta}_{s w}=-b_{s w} \dot{\theta}_{s w}+T_{t b}+u_{\tau} \\
T_{t b}=K_{t b}\left(\theta_{s w}-\theta_{p}\right)
\end{gathered}
$$

where $u_{\tau}$ and $T_{t b}$ are the driver torque and the torque developed due to flexibility of the torsion bar. $\theta_{s w}, J_{s w}$ and $b_{s w}$ are angle of rotation, moment of inertia and viscous damping of the steering column.

The rack and its connection to the wheel spindle as well as intermediate shaft of steering are combined together, and represented as a single inertia at the intermediate shaft. The dynamics of the steering intermediate shaft (pinion) are described by:

$$
J_{p} \ddot{\theta}_{p}=-K_{p} \theta_{p}-b_{p} \dot{\theta}_{p}+T_{t b}+T_{a}+T_{S A T}+u_{d}
$$

where $\theta_{p}, J_{p}$ and $b_{p}$ are angular displacement, inertia and damping of the steering system at the pinion. $K_{p}$ is the stiffness induced by the inclined kingping axis on the rack displacement. $T_{S A T}$ and $u_{d}$ represent the self-aligning torque (SAT) and external torque due to road irregularities or disturbances at the intermediate shaft, respectively. $T_{a}$ is the assist torque provided by the DC motor; assuming the worm gear ratio of $G, T_{a}=G T_{e}$ (see Eq. (2)).

A single-track or "bicycle" model [30], as shown in Fig. 3, can be used to analyze the vehicle dynamics behavior. This model includes several important exclusions and simplifications. These simplifications greatly reduce the model's complexity and degrees of freedom, but do not significantly affect the vehicle lateral dynamics. For example, in the bicycle model, the height of vehicle's center of gravity is at the road surface; therefore, the roll and pitch angles of the vehicle are neglected. As a result, the normal tire force at the left and right sides of vehicle remain the same during cornering, so in this model, the left and right wheels are lumped together and represented by a single wheel. In this section, a bicycle model is used to capture the lateral dynamics of the vehicle and estimate the self-aligning torque of front wheels. The vehicle's velocity at the center of mass is denoted by $V$ and makes an angle $\beta$ with the heading direction of the vehicle. The side slip angle $(\beta)$ and yaw rate $\left(\omega_{z}=\dot{\psi}\right)$ of the vehicle's center of mass are selected as the state variables of the bicycle model. The equations of motion of this model are expressed as follows:

$$
\begin{gathered}
m v_{x}\left(\dot{\beta}+\omega_{z}\right)=F_{y f}+F_{y r} \\
I_{z z} \dot{\omega}_{z}=L_{f} F_{y f}-L_{r} F_{y r}
\end{gathered}
$$

where $F_{y f}$ and $F_{y r}$ are front and rear lateral force of the wheels and are approximated by a linear tire model, in contrast to the Fiala tire model $[31 ; 32]$ used in the high-fidelity model:

$$
\begin{aligned}
& F_{y f}=C_{\alpha f} \alpha_{f} \\
& F_{y r}=C_{\alpha r} \alpha_{r}
\end{aligned}
$$

As shown in Fig. 3, the front and rear slip angles with small steer angles can be approximated as follows:

$$
\begin{gathered}
\alpha_{f}=\frac{v_{y}+L_{f} \omega_{z}}{v_{x}}-\delta_{f} \\
\alpha_{r}=\frac{v_{y}-L_{f} \omega_{z}}{v_{x}}
\end{gathered}
$$




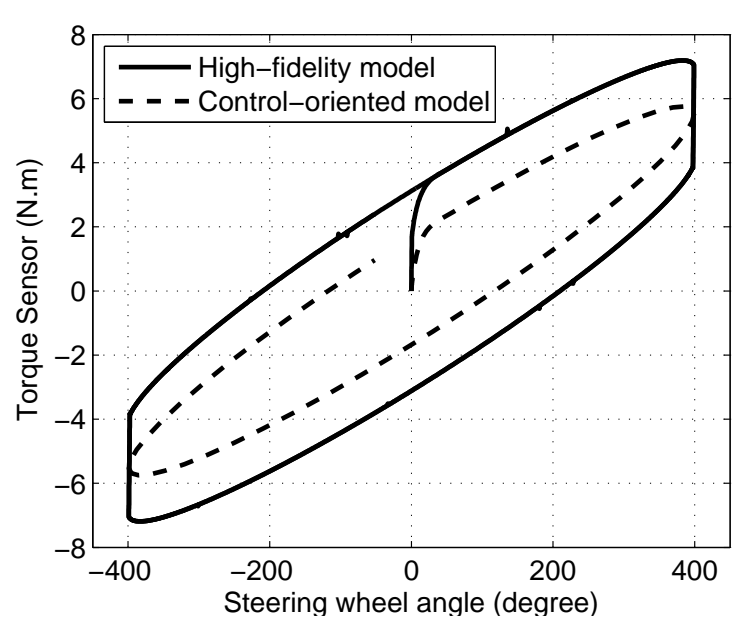

(a)

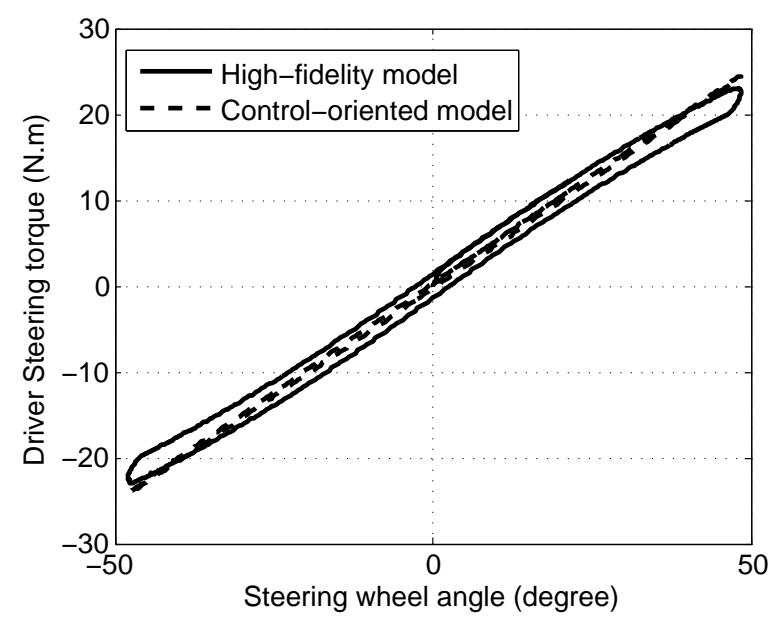

(b)

Fig. 4. (a) The steering torque required versus steering wheel motion when the front wheels are on the friction-less pads, (b) The steering torque required for a smooth steering wheel motion at $10 \mathrm{~m} / \mathrm{s}$ vehicle speed when EPS is off

In these equations, the longitudinal velocity of the vehicle at center of mass is assumed constant and is expressed as $v_{x}=V \cos \beta$, and the steering angle of front wheel is represented by $\delta_{f}$.

Self-aligning torque (SAT) is a nonlinear function of the wheel's slip angle. However, for small slip angles, the SAT can be approximated with a linear function of slip angle $\left(\alpha_{f}\right)$. Assuming the steering angle of the front wheels is $\delta_{f}=\theta_{p} / G_{\text {steering }}$, the SAT can be found from Eq. (13). $G_{\text {steering }}$ is the ratio of the rotation of steering wheel angle to the average value of left and right wheel steer angles.

$$
T_{S A T}=C_{T \alpha} \alpha_{f}=C_{T \alpha}\left(\delta_{f}-\beta-\frac{L_{f} \omega_{z}}{v_{x}}\right)
$$

The remaining parameters are defined and summarized in Table. 1 of Appendix B. The control-oriented model consists of four linear ordinary differential equations (ODEs), three of which are second-order, which is equivalent to a set of seven first-order linear ODEs. The state-space representation of the control-oriented model is presented in Appendix A.

\subsection{Model Validation}

To use the aforementioned control-oriented model in the control design process, the model should be a good representation of the actual system. To demonstrate this, a sinusoidal steering maneuver is simulated for the control-oriented and the highfidelity models. Figure 4 shows the torque required to steer the wheels against the steering wheel angle in the following situations. Figure $4 \mathrm{a}$ shows the required torque when the wheels are on friction-less pads; this graph is used to identify $b_{p}$ and $K_{p}$ coefficients, while Fig. $4 \mathrm{~b}$ is used to estimate the SAT contribution to the required steering torque. In this simulation, the longitudinal speed of the car is $10 \mathrm{~m} / \mathrm{s}(36 \mathrm{~km} / \mathrm{hr})$. An optimization approach is used to find the steering and vehicle parameters. The discrepancy seen in Fig. 4a is the result of the absence of Coulomb friction in the control-oriented model. This conclusion is verified by removing the Coulomb friction from the steering system and comparing the steering torques. The Coulomb friction is not included in the control-oriented model to keep the linearity of the model.

Then, a random steering maneuver with different voltage and disturbance inputs to the system as shown in Fig. 5a is simulated for the control-oriented and high-fidelity models. Since the maximum driver torque is usually about $10 \mathrm{~N} . \mathrm{m}$, a sinusoidal driver torque input with a magnitude of $10 \mathrm{~N} . \mathrm{m}$ is used. The magnitude of the terminal voltage is assumed to be $5 \mathrm{~V}$, and the magnitude of the disturbance force at the rack is $500 \mathrm{~N}$. Figure 5 shows the steering wheel angle, torque sensor and lateral acceleration of the high-fidelity vehicle model and the tuned control-oriented model. These states are selected 


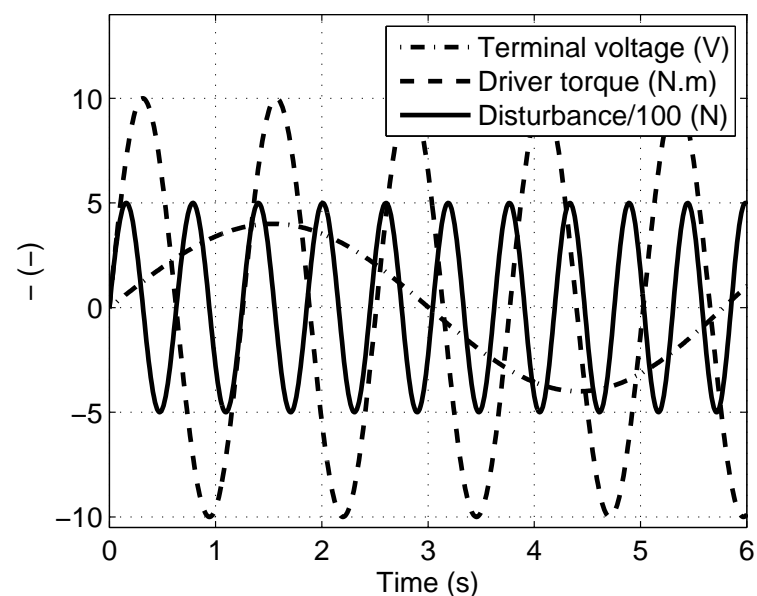

(a)

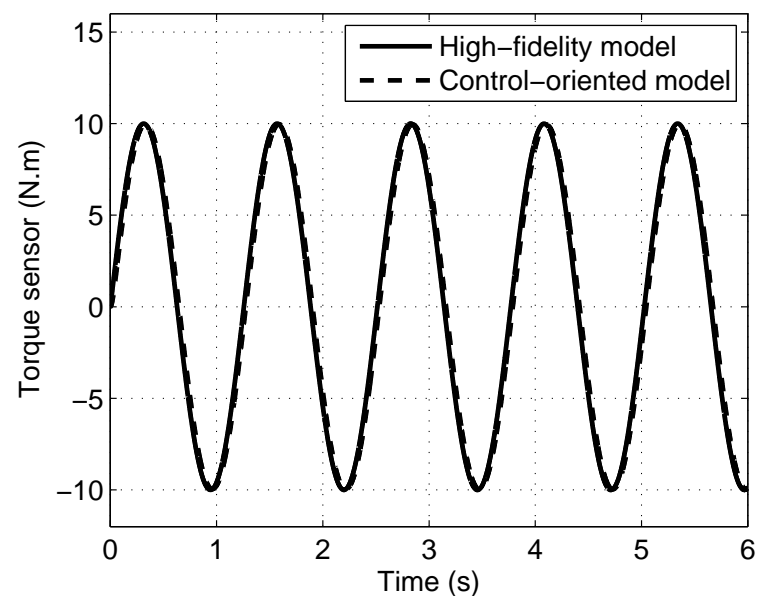

(c)

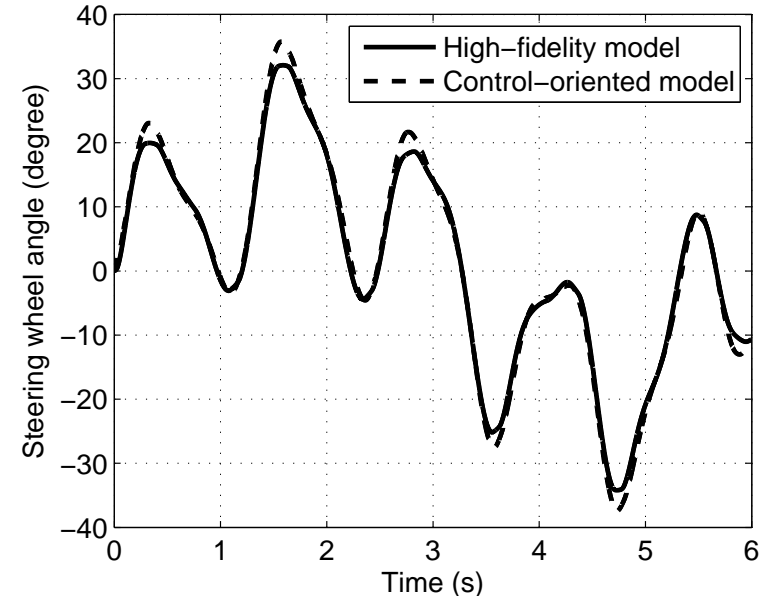

(b)

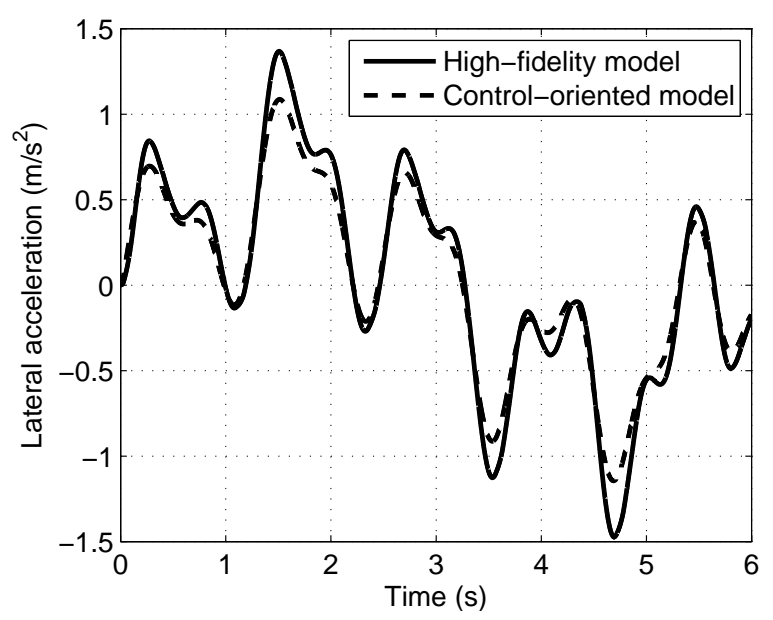

(d)

Fig. 5. Comparison of the control-oriented model with the high-fidelity vehicle model (a) Inputs to the system: terminal voltage, driver torque and disturbance force at the rack, (b) Steering wheel angle, (c) Torque sensor (torsion bar torque), (d) Vehicle lateral acceleration

since they are the sensor measurements of the EPS system, and they should agree well with the real measurements (in this case the high-fidelity model). The results show a good correlation between the models. As shown in Fig. 5c, the torque sensor value for all models is the same since the dynamics between the steering input and torque sensor measurement are closely modeled in both models. However, other measurements have slight differences since the vehicle dynamics is simplified in the control-oriented model.

\section{Optimal Model-Based Controller}

Model-based control design is now widely accepted by the automotive industry as a time-saving and cost-effective approach because it enables optimal application of available mathematical-physical knowledge to achieve complex objectives. Compared to static rule-based control, this approach has better performance in the transient response of the vehicle. However, challenges are still posed in practical applications.

In this research, to investigate the application of optimal control to electric power steering systems, an observer-based optimal disturbance rejection controller (modified Linear Quadratic Gaussian - LQG) is presented. 
As shown in Fig. 6, the steering/vehicle system is subjected to two exogenous inputs, the driver steering torque $\left(u_{\tau}\right)$ and disturbance torque $\left(u_{d}\right)$, and a known input of the electric motor voltage $\left(u_{v}\right)$. The EPS controller should reject or attenuate any unwanted oscillations on the steering wheel while magnifying the driver's steering torque (based on the EPS characteristic curves). Since the driver's steering torque is unknown to the LQG controller, here, the torque sensor value is used instead of steering torque in the EPS control logic. Therefore, the number of unknown inputs for the controller is reduced to one.

Since not all states of the control-oriented model are measured using the external sensors, some states should be reconstructed using the available measurements and by use of an observer (Kalman filter). The difference between real (y) and estimated $(\hat{\mathbf{y}})$ value of sensor measurements due to external disturbances and different initial conditions are fed back to the observer (through an observer gain $(L)$ ) to adjust the state estimations $(\hat{\boldsymbol{x}})$ and to identify the external disturbance $\left(\hat{u}_{d}\right)$.

The disturbance rejection observer uses an augmented disturbance model to estimate the states and unknown disturbance. The observer predicts the unknown disturbance using the difference between the estimated output and actual measurements. Since the driver torque and EPS electric motor current are measured by sensors, sudden changes in the driver steering torque and EPS control voltage are considered to be valid inputs, not disturbances.

Optimal disturbance rejection can be achieved by including a feedforward controller, canceling the effects of the disturbance, into the control logic [33]. Since the external disturbances to the steering systems are unknown, and it is not possible to predict the exact value of a random process beforehand, this method is impractical. However, an effective disturbance rejection can be achieved by including the dynamic properties of the disturbance in the observer and controller design $[34 ; 35]$. In this research, the resultant torque at the pinion due to road excitations is modeled as a zero-mean colored stochastic process. A shaping filter has been used to transform white noise $w$ into an appropriately stationary random process of disturbance torque $\left(\hat{u}_{d}\right)$. This linear shaping factor is defined as follows:

$$
\begin{aligned}
& \dot{x}_{D}=A_{D} x_{D}+B_{D} w \\
& \hat{u}_{d}=C_{D} x_{D}+D_{D} w
\end{aligned}
$$

where $x_{D}$ is the disturbance state, and $A_{d}, B_{d}, C_{d}$ and $D_{d}$ are shaping filter coefficients. Note that the shaping filter corresponding to Eqs. (14) and (15) is a causal first order low-pass filter with $D_{D}=0$.

By substituting Eq. (15) into the state-space representation of the system (Eq. (38) in Appendix A), the new system can be represented as follows:

$$
\begin{aligned}
& \dot{\boldsymbol{x}}=A \boldsymbol{x}+B_{v} u_{v}+B_{\tau} u_{\tau}+B_{d} u_{d} \\
& =A \boldsymbol{x}+B_{v} u_{v}+B_{\tau} u_{\tau}+B_{d} C_{D} x_{D}+B_{d} D_{D} w
\end{aligned}
$$

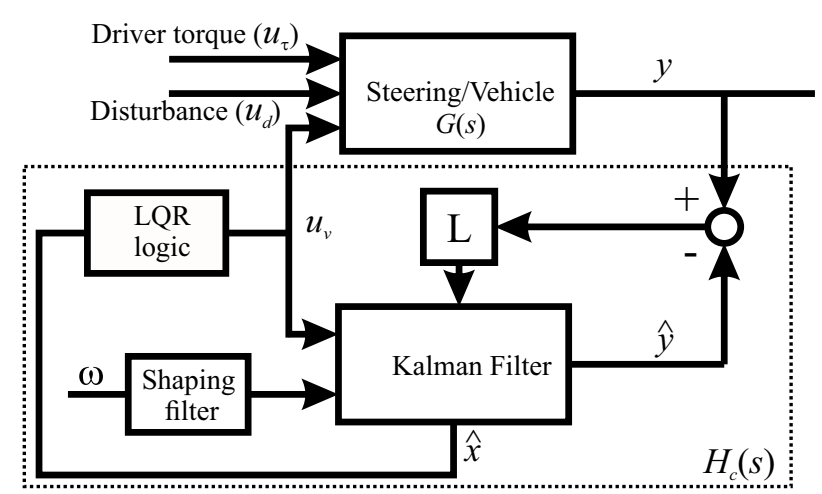

Fig. 6. Workflow of the Linear Quadratic Gaussian controller 
where $\boldsymbol{x} \in \mathbb{R}^{7 \times 1}$ is the state and $A, B_{v}, B_{\tau}, B_{d}$ and $C$ are respectively the system, input and output matrices, and the inputs to the system are defined as driver torque $\left(u_{\tau}\right)$, terminal voltage of electric motor $\left(u_{v}\right)$ and disturbance torque $\left(u_{d}\right)$ at the pinion.

Equation (16) can be rewritten by forming an augmented state vector $\underline{x}$ including the system states $\boldsymbol{x}$ and the disturbance state $x_{D}$ such that the dynamics of the system are described by:

$$
\underline{\dot{\boldsymbol{x}}}=\underline{A} \underline{\boldsymbol{x}}+\underline{B}_{v} u_{v}+\underline{B}_{w} w
$$

where

$$
\underline{\boldsymbol{x}}=\left[\begin{array}{c}
\boldsymbol{x} \\
x_{D}
\end{array}\right], \quad \underline{A}=\left[\begin{array}{cc}
A & B_{u} C_{D} \\
0 & A_{D}
\end{array}\right] \quad \underline{B}_{u}=\left[\begin{array}{c}
B_{v} \\
0
\end{array}\right], \quad \underline{B}_{w}=\left[\begin{array}{c}
B_{u} D_{D} \\
B_{D}
\end{array}\right]
$$

Since the torque sensor value is being used instead of the driver torque in the EPS control logic, the driver torque is removed from the control inputs. In the observer (Kalman filter) design, since the steering wheel angle measurement is available, the driver steering torque (Eq. (4)) has been removed and the steering wheel angle is assumed to be the input to the system. Therefore, the state variables required for estimation are reduced to $\hat{x}=\left[\begin{array}{lllll}\beta & i & r & \theta_{p} & \dot{\theta}_{p}\end{array}\right]^{T}$ and the system is subjected to only one external unknown input (disturbance torque). The state space representation of the system used in the Kalman Filter is as follows:

$$
\begin{gathered}
\dot{\hat{\boldsymbol{x}}}=\hat{A} \hat{\boldsymbol{x}}+\hat{B}_{u} u_{v}+\hat{B}_{\theta} \theta_{s w}+\hat{B}_{d} u_{d} \\
\hat{y}=\hat{C} \hat{\boldsymbol{x}}
\end{gathered}
$$

By substitution of Eq. (15) into Eq. (19) and forming an augmented state vector $\underline{\hat{x}}$ including the system states $\hat{\boldsymbol{x}}$ and the disturbance state $x_{D}$, the dynamics of the Kalman filter system are described by:

$$
\begin{gathered}
\underline{\dot{\boldsymbol{x}}}=\underline{\hat{A}} \underline{\hat{\boldsymbol{x}}}+\underline{\hat{B}}_{u} u_{v}+\underline{\hat{B}}_{\theta} \theta_{s w}+\underline{\hat{B}}_{w} w \\
\hat{y}=\underline{\hat{C}} \underline{\hat{\boldsymbol{x}}}
\end{gathered}
$$

where

$$
\begin{aligned}
& \underline{\hat{\boldsymbol{x}}}=\left[\begin{array}{c}
\hat{\boldsymbol{x}} \\
x_{D}
\end{array}\right], \quad \underline{\hat{A}}=\left[\begin{array}{cc}
\hat{A} & \hat{B}_{d} C_{D} \\
0 & A_{D}
\end{array}\right], \quad \underline{\hat{B}}_{u}=\left[\begin{array}{c}
\hat{B}_{d} \\
0
\end{array}\right], \\
& \underline{\hat{B}}_{\theta}=\left[\begin{array}{c}
\hat{B}_{\theta} \\
0
\end{array}\right], \quad \underline{\hat{B}}_{w}=\left[\begin{array}{c}
\hat{B}_{d} D_{D} \\
B_{D}
\end{array}\right] \quad \underline{\hat{C}}=\left[\begin{array}{ll}
\hat{C} & 0
\end{array}\right]
\end{aligned}
$$

According to the state equations defined in Eq. (20), a Kalman Filter is constructed as an optimal state estimator as follows [33]:

$$
\underline{\dot{\hat{\boldsymbol{x}}}}=\underline{\hat{A}} \underline{\hat{\boldsymbol{x}}}+\underline{\hat{B}}_{u} u_{v}+\underline{\hat{B}}_{\theta} \theta_{s w}+L(\mathbf{y}-\underline{\hat{C}} \hat{\mathbf{x}})
$$

where

$$
L=\Phi \underline{\hat{C}}^{T} V_{2}^{-1}
$$

and $V_{2}$ is the intensity of the sensor noise and $\Phi$ is the solution to the following Riccati equation.

$$
\Phi \underline{\hat{A}}^{T}+\underline{\hat{A}} \Phi+V_{1}-\Phi \underline{\hat{C}}^{T} V_{2}^{-1} \underline{\hat{C}} \Phi=0
$$

where $V_{1}$ is the intensity matrix of the plant disturbance, which is determined by trial and error.

Based on the separation principle, the optimal control can be determined by feeding the estimated states from the Kalman filter into an optimal state feedback controller. Therefore, the optimal control can be found by rearranging the 
estimated states and augmenting the known steering wheel angle and velocity to the state vector estimation,

$$
u_{v}=\underline{K}_{F B}\left[\hat{\boldsymbol{x}}^{T} \theta_{s w} \dot{\theta}_{s w} \hat{u}_{d}\right]^{T}
$$

where

$$
\underline{K}_{F B}=R^{-1} \underline{\hat{B}}_{u}^{T} P
$$

and $R$ is a input usage weight (see Eq. (29)) and $P$ the solution to the following Riccati equation:

$$
P \underline{\hat{A}}+\underline{\hat{A}}^{T} P+Q-P \underline{\hat{B}}_{u} R^{-1} \underline{\hat{B}}_{u}^{T} P=0
$$

where $Q$ is a state deviation weighting matrix (see Eq. (29)).

It is known that the optimal disturbance rejection can be achieved by a full state feedback controller in conjunction with a feedforward controller with a known disturbance. Therefore, it can be seen that by partitioning the feedback gains of the proposed controller into $\underline{K}_{F B}=\left[K_{F B} \mid K_{F F}\right]$, the observer-based disturbance rejection controller can have a similar property; it has a feedback function of system states and feedforward function of the estimation of disturbance, as follows:

$$
u_{v}=\left[K_{F B} \mid K_{F F}\right]\left[\hat{\boldsymbol{x}}^{T} \theta_{s w} \dot{\theta}_{s w} \mid \hat{u}_{d}\right]^{T}=K_{F B}\left[\hat{\boldsymbol{x}}^{T} \theta_{s w} \dot{\theta}_{s w}\right]^{T}+K_{F F} \hat{u}_{d}
$$

The LQG control finds the optimal state feedback gains such that the following cost function is minimized.

$$
J=\int_{0}^{\infty}\left(\underline{\boldsymbol{x}} Q \underline{\boldsymbol{x}}^{T}+\boldsymbol{u} R \boldsymbol{u}^{T}\right) d t
$$

where $Q$ or state deviation weighting matrix is a symmetric positive semi-definite matrix and $R$ or input usage weight is a symmetric positive definite matrix.

In this article, the bi-linear characteristic curve as shown in Fig. 1a is used in the LQG controller. The EPS characteristic curves include two intervals: $\left[0, T_{0}\right)$ is the interval with no steering assistance, and $\left[T_{0}, T_{\max }\right]$ is the interval with linear steering assistance. Therefore, for full tracking of characteristic curves in the whole operating region, two control laws are required. The first law is indicating that current of the electric motor should be zero unless there is a disturbance; in the presence of a disturbance the assist torque should cancel the disturbance torque. Therefore, the following cost function is proposed for the first interval:

$$
J_{1}=\int_{0}^{\infty}\left(q\left(T_{a}-K_{b} \hat{u}_{d}\right)^{2}+\rho u_{v}^{2}\right) d t
$$

where $q$ and $\rho$ are the tracking and input usage weights, and $K_{b}$ is the disturbance rejection feature switch. The disturbance rejection module is turned off when $K_{b}$ is equal to zero, and it is enabled when $K_{b}$ is equal to one.

The second interval suggests that the assist torque be proportional to the torque measured by the torque sensor. To con-

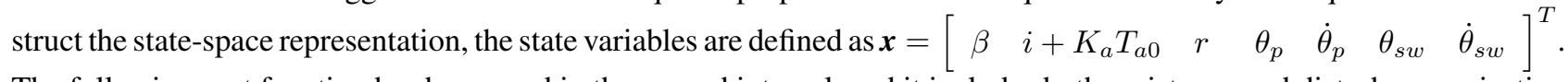
The following cost function has been used in the second interval, and it includes both assistance and disturbance rejection modules:

$$
J_{2}=\int_{0}^{\infty}\left(q\left(T_{a}-T_{a}^{d e s}-K_{b} \hat{u}_{d}\right)^{2}+\rho u_{v}^{* 2}\right) d t
$$

where

$$
T_{a}^{d e s}=K_{a} K_{t b}\left(\theta_{s w}-\theta_{p}\right)
$$




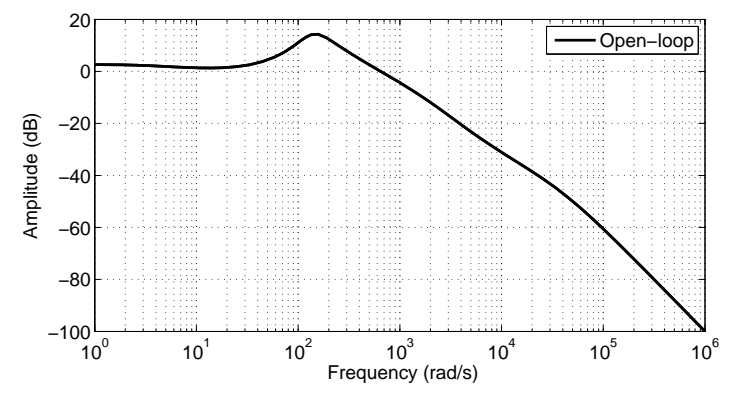

(a)

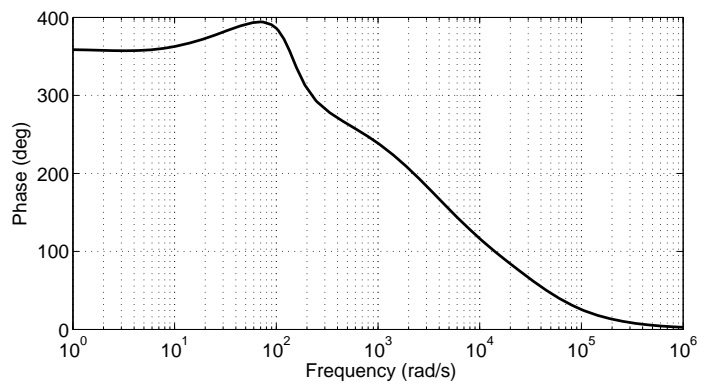

(b)

Fig. 7. Bode plots of the open-loop system with the observer-based disturbance rejection controller (a) Amplitude, (b) Phase

Here, $T_{a}$ and $T_{a}^{\text {des }}$ are actual and desired assist torques and $u_{v}^{*}$ is the modified terminal voltage of the electric motor $\left(u_{v}^{*}=u_{v}+K_{a} R T_{0}\right)$, where $T_{a}^{\text {des }}$ is proportional to the torque sensor value, and $K_{a}$ is the EPS assist gain.

By substituting Eqs. (32) and (2) into Eq. (31) and expanding the equation, the $Q$ and $R$ in their standard integral quadratic form as shown in Eq (29) for the second interval can be described by:

$$
Q=q\left[\begin{array}{cccccccc}
0 & 0 & 0 & 0 & 0 & 0 & 0 & 0 \\
0 & G^{2} K_{e}^{2} & 0 & G K_{a} K_{e} K_{t b} & 0 & -G K_{a} K_{e} K_{t b} & 0 & K_{b} G K_{e} \\
0 & 0 & 0 & 0 & 0 & 0 & 0 & 0 \\
0 & G K_{a} K_{e} K_{t b} & 0 & K_{a}^{2} K_{t b}^{2} & 0 & -K_{a}^{2} K_{t b}^{2} & 0 & K_{b} K_{a} K_{t b} \\
0 & 0 & 0 & 0 & 0 & 0 & 0 & 0 \\
0 & -G K_{a} K_{e} K_{t b} & 0 & -K_{a}^{2} K_{t b}^{2} & 0 & K_{a}^{2} K_{t b}^{2} & 0 & -K_{b} K_{a} K_{t b} \\
0 & 0 & 0 & 0 & 0 & 0 & 0 & 0 \\
0 & K_{b} G K_{e} & 0 & K_{b} K_{a} K_{t b} & 0 & -K_{b} K_{a} K_{t b} & 0 & K_{b}^{2}
\end{array}\right], \quad R=\rho
$$

The weights of current error $(q)$ and terminal voltage $(\rho)$ in cost functions $J_{1}$ and $J_{2}$ are chosen by trial and error to ensure that the current of electric motor will track the desired current with minimum electric motor terminal voltage.

To study the frequency-domain behavior of this controller, an open-loop transfer function of the system including the controller and the linear control-oriented model, as shown in Fig. 6, has been derived. This open-loop transfer function represents the system from the plant control input to the controller output $\left(u_{v}\right)$, and is presented by $H_{u}(s)=H_{c}(s) G(s)$. In this transfer function $G(s) \in \mathbb{R}^{8 \times 1}$ is the control-oriented model transfer function and $H_{c} \in \mathbb{R}^{1 \times 8}$ is the controller transfer function, and they are defined as follows:

$$
\begin{gathered}
G(s)=(s I-A)^{-1} B_{v} \\
H_{c}(s)=\left[\begin{array}{ll}
K_{1} & K_{2}
\end{array}\right] H(s)=\left[\begin{array}{ll}
K_{1} H_{\overline{K F}}(s) & K_{2} H_{\theta_{s w}}(s)
\end{array}\right]
\end{gathered}
$$

where $\left[\begin{array}{ll}K_{1} & K_{2}\end{array}\right]$ are the LQR feedback gains, in which $K_{2}$ affects the steering wheel states and $K_{1}$ affects all the remaining states of the system, $H_{\overline{K F}}(s) \in \mathbb{R}^{6 \times 1}$ represents the Kalman filter, and $H_{\theta_{s w}}(s) \in \mathbb{R}^{2 \times 1}$ represents the measured steering wheel angle and its angular velocity transfer functions.

Figure 7 shows the amplitude and phase responses of the open-loop transfer function from the Bode plot of the system. This figure suggests that the gain margin and phase margin of this controller are 7.65 and $74.6^{\circ}$, respectively. The positive gain and phase margins indicate that there is a large safety margin before the closed-loop system become unstable. 


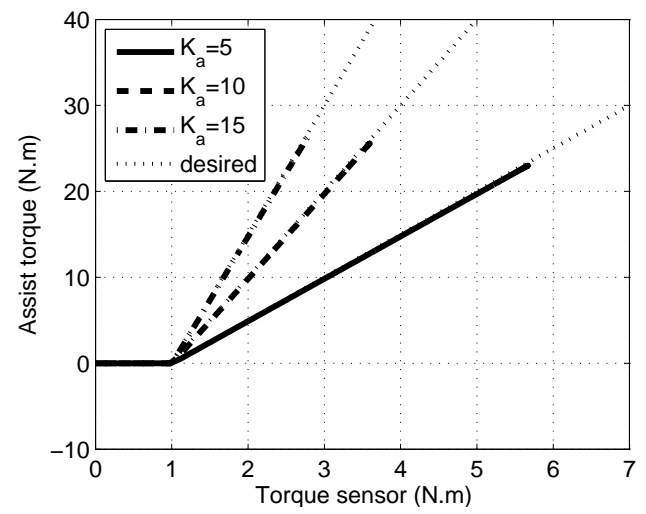

Fig. 8. The produced assist torque versus torque sensor value with different assist gains

\section{Simulation Results and Discussion}

To evaluate the performance of the proposed controller, closed-loop simulations with the high-fidelity vehicle model are required. In this section, the effects of assist gain variation and disturbances on the performance of the controller have been studied. To study the robustness of the EPS controller to different assist gains, a sinusoidal steering maneuver with amplitude of $60^{\circ}$ and frequency of $0.5 \mathrm{rad} / \mathrm{s}$ is performed. In these simulations, the vehicle speed is equal to $10 \mathrm{~m} / \mathrm{s}$ and the length of no-assist zone $\left(T_{a 0}\right)$ is set to $1 \mathrm{~N} . \mathrm{m}$. The Disturbance Rejection (DR) module is switched off $\left(K_{b}=0\right)$ and $K_{a}$ is changed to 5, 10 and 15, consecutively. As shown in Fig. 8 the proposed controller can closely follow the desired assist curves with different assist gains.

Second, to study the performance of the controller in a real-world condition, two maneuvers have been used. In the first maneuver, the vehicle is driving in a straight lane while suddenly a step-like disturbance torque (Fig. 10a) is applied to the steering column. In the second maneuver, the driver performs a sinusoidal slalom-like maneuver, in the presence and absence of a disturbance torque. In both cases, the vehicle speed is equal to $10 \mathrm{~m} / \mathrm{s}$ and the assist gain $\left(K_{a}\right)$ is set to 3 and 10 N.m, respectively.

\subsection{Straight-line Driving Maneuver}

In the first maneuver, while the vehicle is driving in a straight lane, three conditions have been applied to the EPS controller: the EPS system is off, the EPS system is enabled but the DR module is off, and the EPS system is fully functional. As shown in Fig. 9 with the given disturbance, the first situation has the largest steering wheel angle variation while in the second situation, the assistance module enables the driver to produce more resistive torque which results in less steering wheel angle variation. In the third situation, when the DR module is on, the EPS controller estimates the disturbance automatically and produces a counter torque at the steering wheel, so the effects of the disturbance are greatly reduced at the steering wheel.

Fig. 10a shows the performance of the Kalman filter in the estimation of the external disturbance. It should be noted that Coulomb friction between the rack and rack housing is also considered as a disturbance since it is not included in the control-oriented model and it acts as an external force to the rack. Fig. 10b shows the assist torque produced by the EPS controller; it can be seen that the estimated disturbance is effectively used by the disturbance rejection module of EPS controller to reduce the transferred disturbance to the driver's hands. 


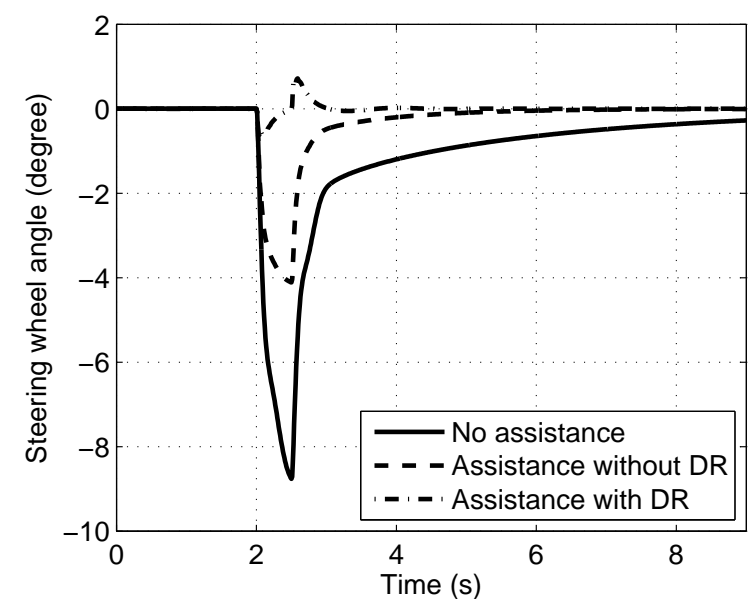

Fig. 9. Comparison of the steering wheel angles in the straight line simulation in presence of a step-like disturbance

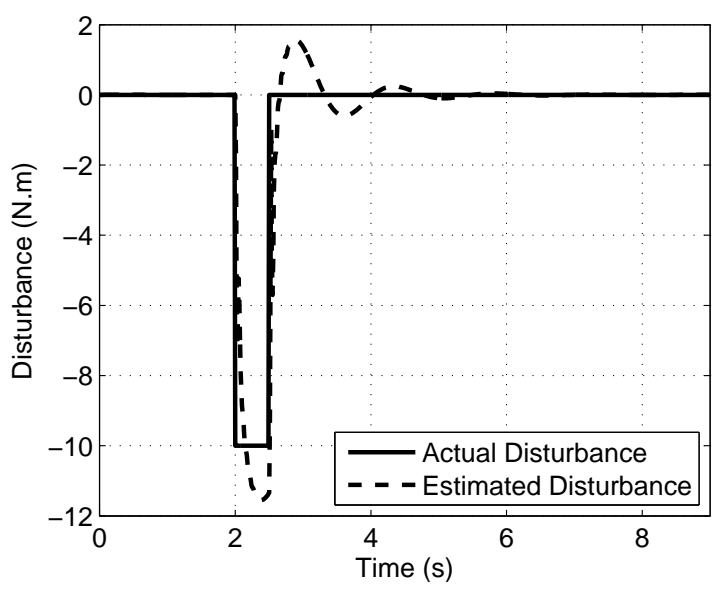

(a)

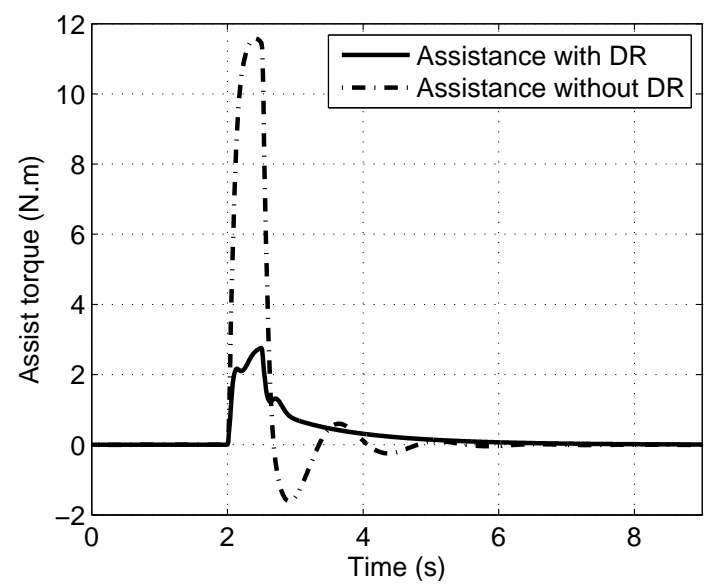

(b)

Fig. 10. (a) Comparison between the actual and estimated value of disturbance, (b) Torque sensor measurement when DR module is on and off 


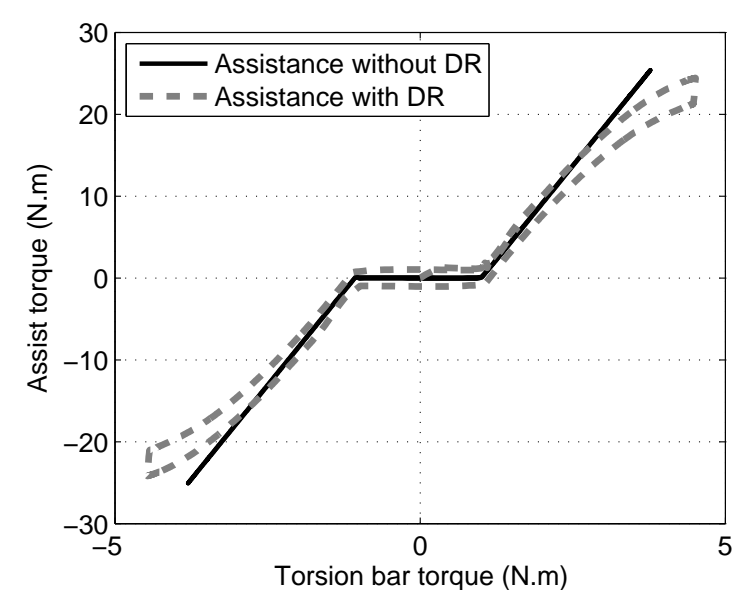

(a)

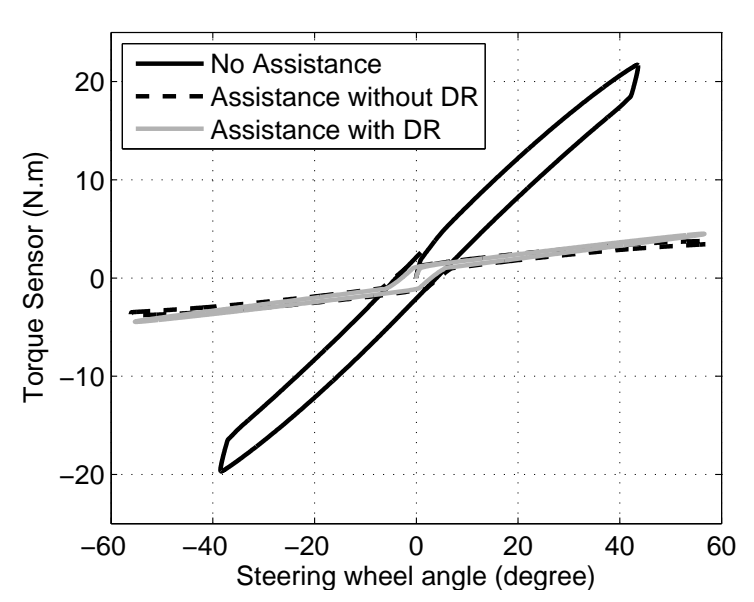

(b)

Fig. 11. Simulation of the proposed EPS controller applied to the high-fidelity vehicle model in a smooth steering maneuver (a) The produced assist torque versus torque sensor value in the absence of external disturbance with and without the DR module, (b) The required steering torque versus the steering wheel position in the absence of external disturbance with and without the DR module

\subsection{Slalom Driving Maneuver}

In the second maneuver, a sinusoidal rotation of steering wheel with frequency of $0.5 \mathrm{rad} / \mathrm{s}$ and amplitude of 50 degrees is performed, and similar situations to the first maneuver have been studied in the presence and absence of an external disturbance.

In the first case, without the existence of any external disturbances, the performance of the controller for all three situations has been studied. As shown in Fig. 11a when the DR module is off, the assist curve is equal to its nominal value (bi-linear curve) as expected; however, when the DR module is enabled, the EPS controller produces more or less assist torque based on the estimated value of disturbance (as shown in Fig. 12a). Since there is no external disturbance to the system, the estimated value represents the friction present in the steering system and the difference between actual self-aligning torque of wheels and the linear response of the control-oriented model.

Figure $11 \mathrm{~b}$ shows the torque sensor (driver steering torque) value against the steering wheel angle (the so called Lissajous curves) for all the aforementioned situations. It can be seen that in the no-assist zone, the Lissajous curves in all the situations are on top of each other, which represents the regular behavior (without EPS) of the steering system. In the linear assist zone, the driver's torque is significantly reduced since the controller is producing the assist torque proportional to the steering torque. A reduction in the area inside the required steering torque curve when the DR module is on in comparison with the control without DR can be observed since the EPS controller is compensating for the friction present. It can be also noted that the EPS controller with DR leads to a linear response while the behavior of the system is nonlinear.

Figure $12 \mathrm{~b}$ shows the torque sensor value in all aforementioned situations. As expected, the steering torque for the case without assistance is higher than the other cases, and the steering torque when the DR module is on is slightly higher than the situation without DR since the controller is enforcing a linear response to the steering system. It should be noted that if the parameters of the vehicle model change from their nominal values, a parameter identification algorithm can be employed to update the EPS controller parameters. However, in the case of a sudden change in the parameters, the controller enforces the linear behavior of the control-oriented model with its nominal parameters.

In the second maneuver, in the presence of an external disturbance, the effectiveness of the DR module in a realistic steering maneuver has been studied. As shown in Fig. 13b, in the situation when the DR module is off, the Lissajous curve is contorted, meaning that the disturbances are transferred to steering wheel and the driver's hands (see fig. 14b). In the third situation (EPS on, DR on), these oscillations are significantly reduced because the EPS controller uses the estimated 


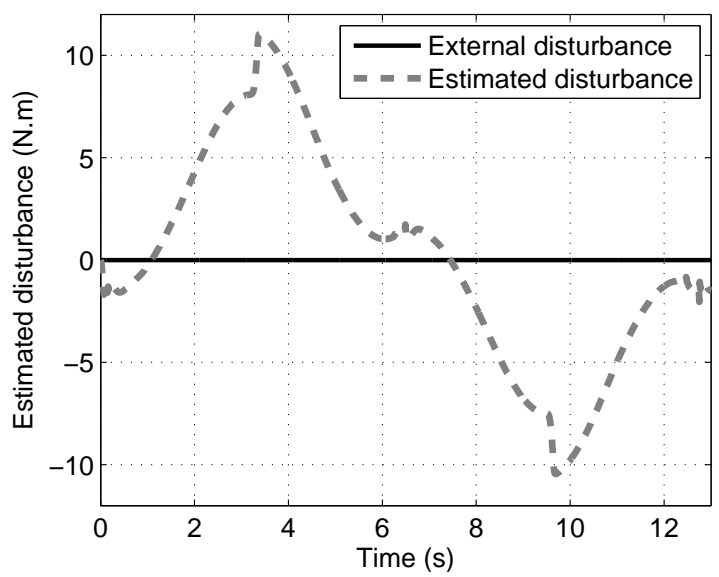

(a)

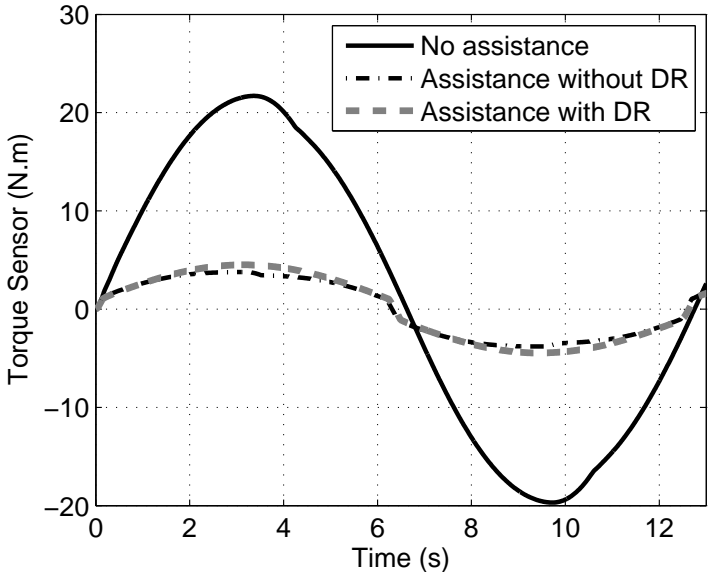

(b)

Fig. 12. (a) Comparison between the actual and estimated value of disturbance, (b) Comparison of the required driver torque with and without EPS controller in a sinusoidal steering maneuver

value of disturbance to attenuate the oscillations on the steering wheel. As shown in Fig. 13a, the produced assist torque, when the DR module is on, is highly nonlinear while the assist torque when the DR is off is bi-linear.

Fig. 14a shows the estimated value of disturbance in comparison with the external disturbance; similar to Fig. 12a, it should be noted that the friction forces are also included in the estimated value of disturbance. Figure 14b shows the torque sensor value for all aforementioned situations. In the case with enabled DR module, the disturbance is successfully attenuated and the behavior of the system is linear.

\subsection{Slalom Driving Maneuver with Measurement Noise}

Kalman filters can remove noise from measurement signals by considering a predefined model of the measurements. In this research, a similar scenario to the sinusoidal steering input with corrupted measurement signals are studied. Fig. 15 shows the corrupted and reconstructed torque sensor and yaw rate values. It can be seen that the reconstructed signals are noise-free and conveying the correct dynamics of the system.

As shown in Fig. 16, the performance of the controller (when the DR module is on and in the presence of measurement noise) is not degraded in comparison with the case without measurement noise.

Since the steering wheel angle is the input to the observer, the steering wheel angle is left noise free; however, in the case of a corrupted steering wheel angle measurement, a separate filter should be designed for this signal.

\section{4. $H_{2} / H_{\infty}$ multi-objective EPS controller}

In this section, the proposed controller is compared to a multi-objective $H_{2} / H_{\infty}$ EPS controller, which represents the current state-of-the-art in EPS system control [14; 36; 37], and a proportional-derivative-integral (PID) controller [22]. The PID controller provides assistance based on the predefined bi-linear characteristic curves, and there is no disturbance rejection module included in this controller. The multi-objective $H_{2} / H_{\infty}$ control strategy improves the transient behavior of the $H_{\infty}$ feedback system by forcing the closed-loop poles of the system to stay in a specified region. In other words, this controller minimizes an $H_{2}$ norm of a performance index, while the $H_{\infty}$ norm of another performance index is less than a prescribed band $\left(\gamma_{1}\right)$ as shown in Eq. (36). This makes the multi-objective $H_{2} / H_{\infty}$ controller desirable in EPS systems, since it can provide accurate assistance to the driver (using $\mathrm{H}_{2}$ ) and at the same time reduces the external disturbances 


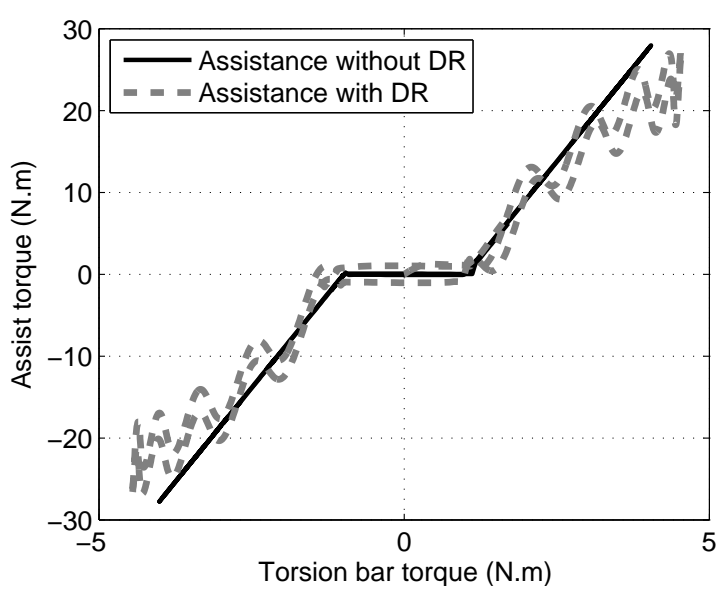

(a)

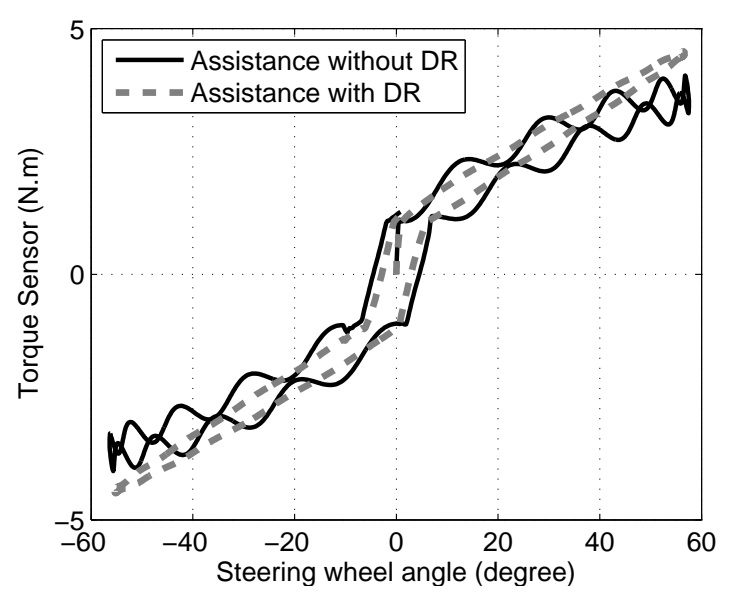

(b)

Fig. 13. Simulation of the proposed EPS controller applied to the high-fidelity vehicle model in a smooth steering maneuver (a) The produced assist torque versus torque sensor value in the presence of external disturbance with and without the DR module, (b) The required steering torque versus the steering wheel position in the presence of external disturbance with and without the DR module

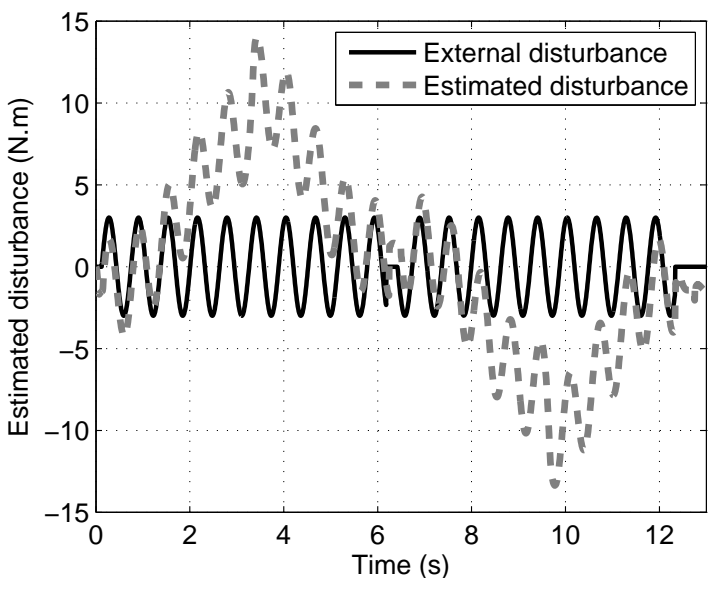

(a)

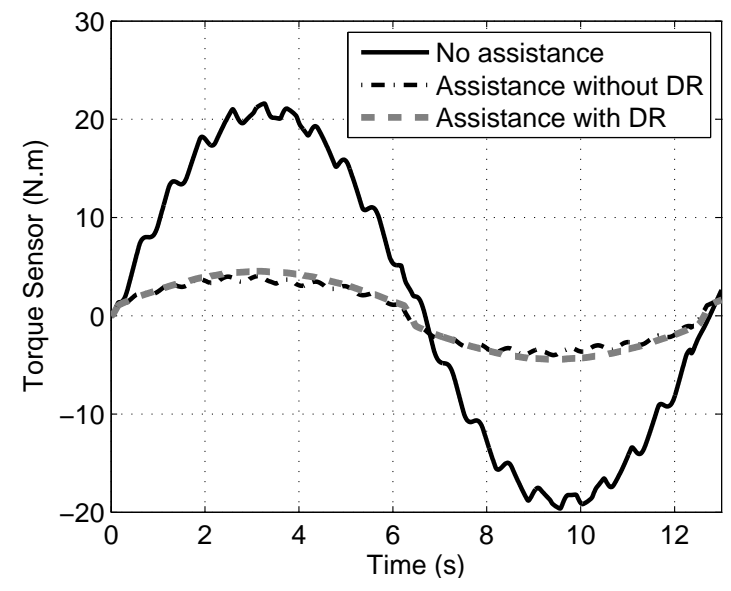

(b)

Fig. 14. (a) Comparison between the actual and estimated value of disturbance, (b) The required steering torque with and with out the proposed EPS controller 


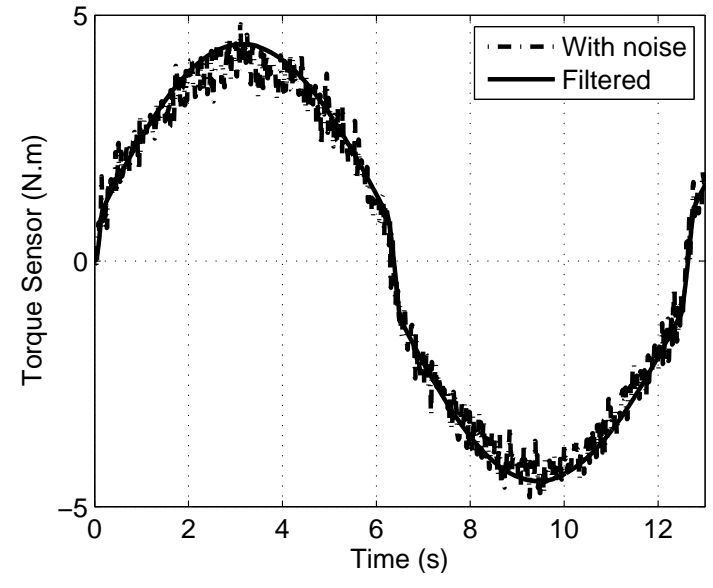

(a)

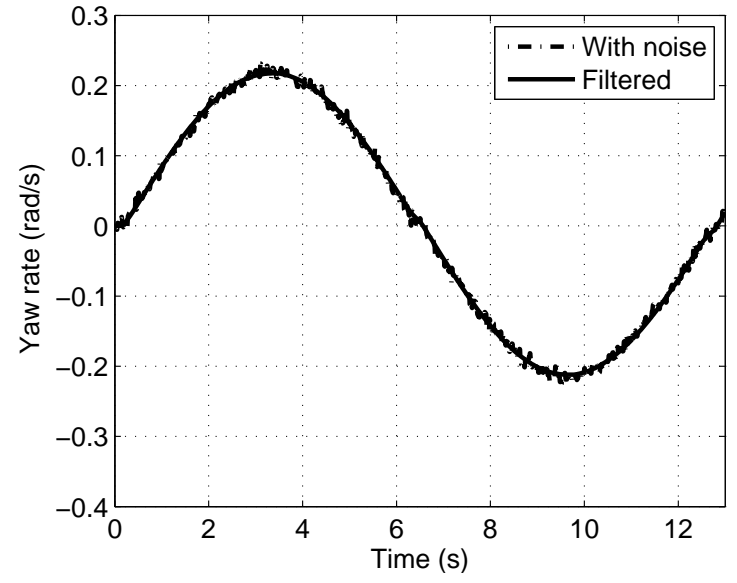

(b)

Fig. 15. Corrupted and reconstructed measurement signals (a) Torque sensor, (b) Vehicle yaw rate

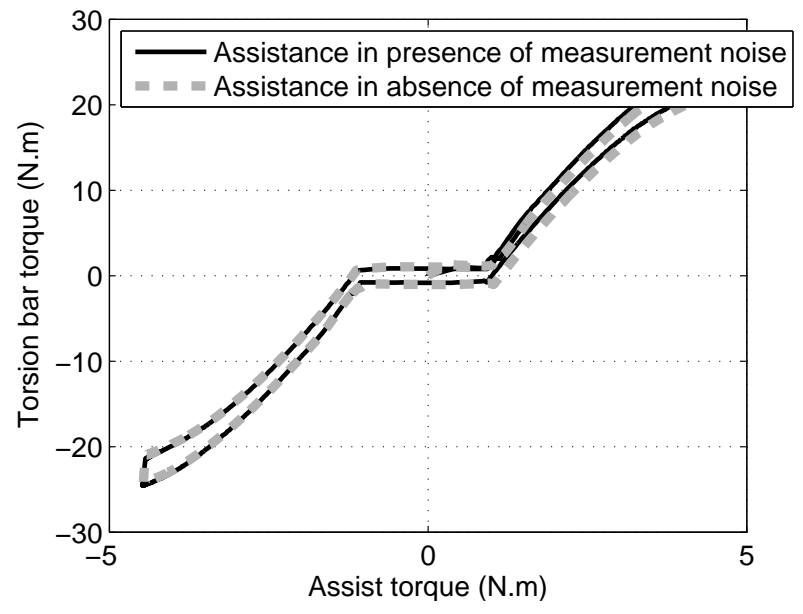

(a)

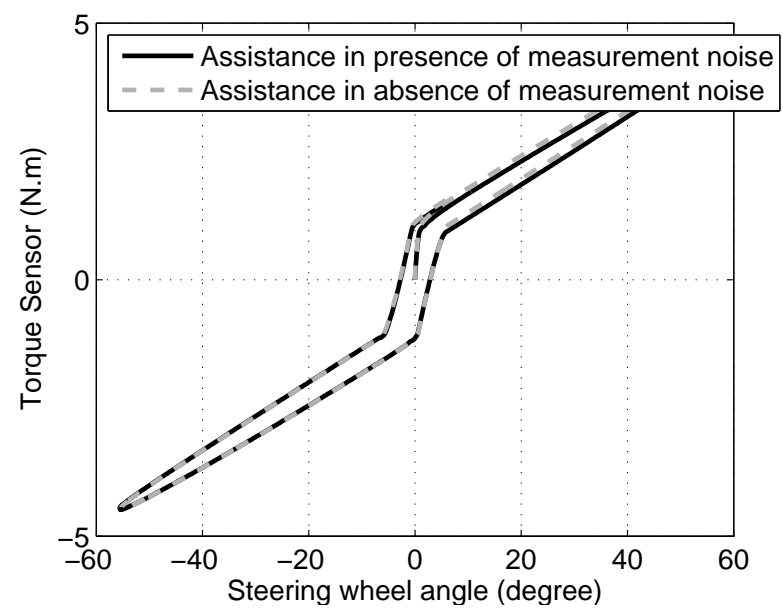

(b)

Fig. 16. Simulation of the proposed EPS controller applied to the high-fidelity vehicle model in a smooth steering maneuver (a) The produced assist torque versus torque sensor value in the presence of measurement noise with the DR module, (b) The required steering torque versus the steering wheel position in the presence of measurement noise with the DR module 


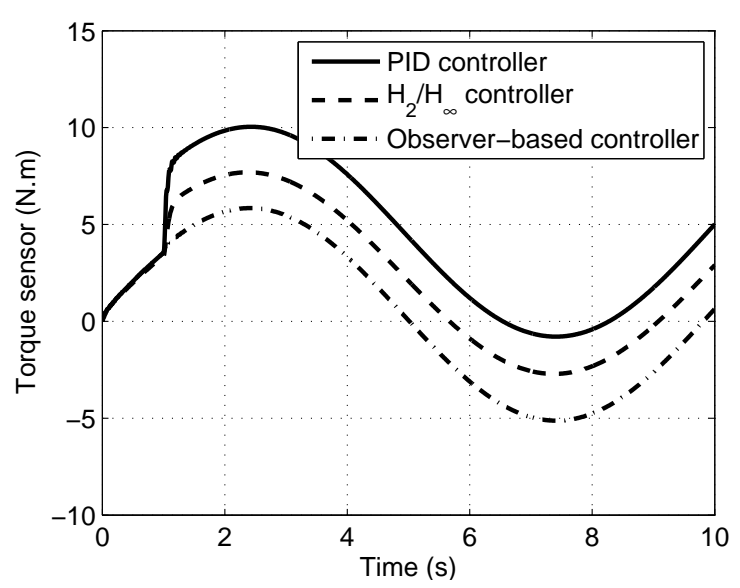

(a)

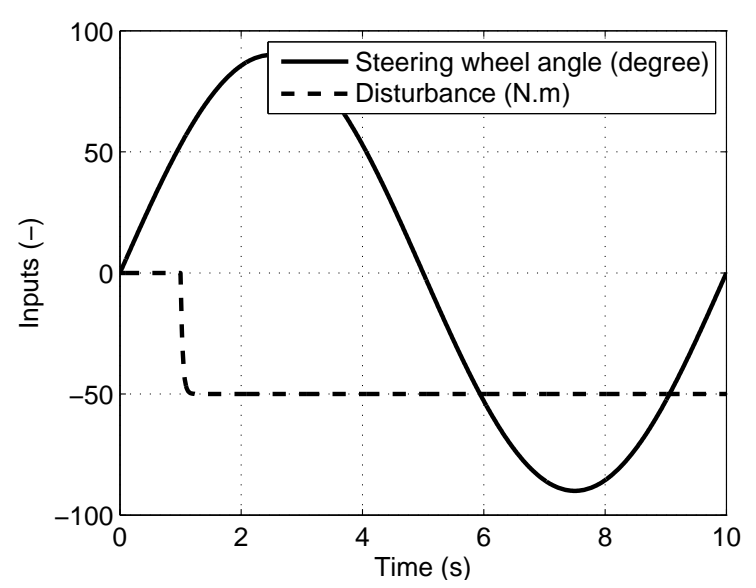

(b)

Fig. 17. (a) The torque sensor measurements in presence of an external disturbance for three different controllers, (b) The step-like external disturbance and the steering wheel angle variation

or measurement noise on the system (using $H_{\infty}$ ). Generally, an $H_{2} / H_{\infty}$ output-feedback EPS controller in the form of $u_{v}=K \boldsymbol{y}$ can be found if there is a $\gamma_{1}>0$ such that the following is satisfied [38].

$$
J=\left\{\begin{array}{c}
\left\|T_{z_{\infty} \omega}\right\|<\gamma_{1} \\
\min \left\|T_{z_{2} \omega}\right\|
\end{array}\right.
$$

where $T_{z_{\infty} \omega}$ and $T_{z 2 \omega}$ are closed-loop transfer functions from the disturbance inputs $\left(\boldsymbol{\omega} \in \mathbb{R}^{2}\right.$ ) to the controlled outputs $z_{\infty}$ and $z_{2}$, respectively. The generalized state-space representation of the open-loop system, in our case, the EPS system, can be described as follows:

$$
\left\{\begin{array}{c}
\dot{\boldsymbol{x}}=A \boldsymbol{x}+\left[B_{\tau} B_{d}\right] \boldsymbol{\omega}+B_{v} u_{v} \\
z_{\infty}=C_{1} \boldsymbol{x}+D_{11} \boldsymbol{\omega}+D_{12} u_{v} \\
\boldsymbol{z}_{2}=C_{2} \boldsymbol{x}+D_{21} \boldsymbol{\omega}+D_{22} u_{v} \\
\boldsymbol{y}=C \boldsymbol{x}+D_{y 1} \boldsymbol{\omega}+D_{y 2} u_{v}
\end{array}\right.
$$

where $\boldsymbol{x} \in \mathbb{R}^{7 \times 1}$ is the state, $A, B_{\tau}, B_{d}, B_{u}, C_{1}, C_{2}, D_{11}, D_{12}, D_{21}, D_{22}, C, D_{y 1}, D_{y 2}$ are the system matrices, and $z_{2}$, $z_{\infty}, \boldsymbol{y}$ are controlled outputs and available measurements to the controller. In this article, the controlled outputs are chosen similar to the outputs shown in [37; 14], where $z_{2}=\left\{\begin{array}{c}W_{1}\left(T_{a}-K_{a} T_{d}\right) \\ W_{2}\left(u_{v}\right)\end{array}\right\}$ and $z_{\infty}=W_{3}\left(T_{a}-K_{f} T_{S A T}\right)$. Here, to be consistent with the observer-based EPS controller, similar measurements are available to the controller, and $K_{f}$ is assumed to be zero to provide similar road feel. The weight functions are selected as $W_{1}=\frac{1}{s / 15+1}, W_{2}=0.0001$ and $W_{3}=\frac{s}{s+100}$.

Figure 17 shows the torque sensor readings of the control-oriented model equipped with multi-objective $H_{2} / H_{\infty}$ controller, observer-based disturbance rejection controller, and PID controller during a sinusoidal steering maneuver. In this maneuver, a step-like disturbance is applied at the pinion one second after the driver begins performing the sinusoidal steering as shown in Fig. 17b. The PID controller transfers a large part of the disturbance to the steering wheel in comparison to the other controllers as shown in Fig. 17a, since there is no disturbance rejection module in this controller. The $H_{2} / H_{\infty}$ controller reduces the effect of the external disturbance on the steering column since it minimizes the maximum effects of the disturbance on the steering column torque; however, this controller cannot completely isolate the expected steering response from the unknown external disturbance. On the other hand, the observer-based EPS controller can accurately estimate the disturbance applied on the steering column and attenuate the disturbance by providing an opposite torque 


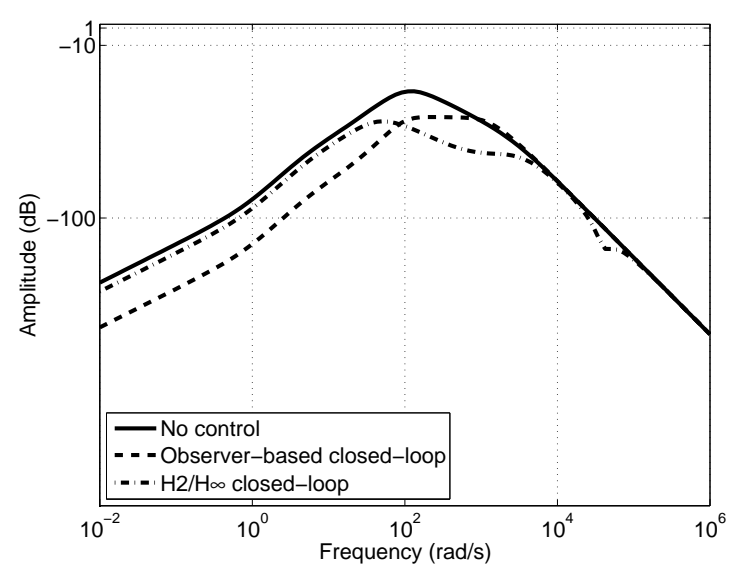

(a)

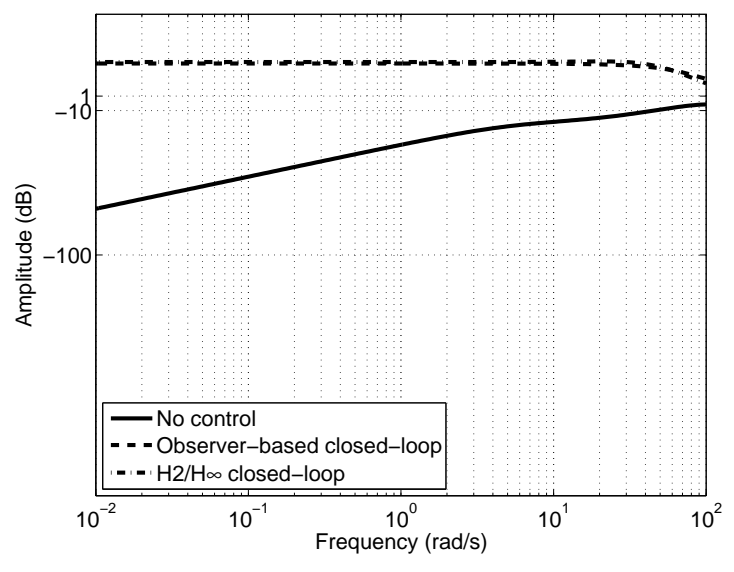

(b)

Fig. 18. Bode plots of the closed-loop system with the observer-based disturbance rejection controller (a) the transfer function with disturbance torque at the pinion as the input and torque sensor measurement as the output, (b) the transfer function with driver steering torque as the input and EPS assist torque as the output

at the steering column. Therefore, the observer-based disturbance rejection controller has superior disturbance rejection properties to the other two contestants.

Figure 18 shows the closed-loop frequency responses of the observer-based disturbance rejection and the $H_{2} / H_{\infty}$ EPS controllers on the control-oriented model. Figure 18a shows the frequency responses of the system by considering the disturbance as the input and steering column torque (torque sensor) as the output. As shown in Fig. 18a, the magnitude of the response is highly reduced, which means that the controller can effectively reduce the low and high frequency disturbance. Similarly, the frequency responses of the closed-loop system considering the driver steering torque as the input and EPS assistance as the output is shown in Fig. 18b. As expected, the assistance module in the EPS controllers results in an increase in the steering assist over the entire frequency range.

\section{Conclusions and Future Work}

In this paper, an observer-based disturbance rejection controller for electric power steering systems is proposed. To construct the EPS controller, the LQG control approach with separate observer and controller has been used. A Kalman filter augmented with a shaping filter is used to estimate the unmeasured states and the external disturbances to the steering system. Simultaneously, the estimated states and the disturbance is used in the LQR controller with a new formulation of control objective function to actively cancel the disturbances while magnifying the driver's steering torque.

Then, the proposed EPS controller is evaluated in a software-in-the-loop simulation using a high-fidelity vehicle model to ensure the robust performance of the controller in a real-world condition. In the case with no external disturbance to the system, the disturbance observer estimates the steering's component frictions, which is deviating the system states from their linear response. Similarly, in the presence of an external disturbance, the identified disturbance is the combination of friction and external disturbance. Removing this identified disturbance from the system reduces the driver torque, which is enforcing the linear response of control-oriented model to the system. Therefore, the proposed EPS controller improves the steering feel by reducing the required steering torque and reducing the oscillations due to road irregularities.

The proposed modified LQG controller has strong robustness against measurement noise and external disturbances. The simulation results show that it can efficiently attenuate the interference caused by road random excitation and can competently work with the corrupted measurements. Based on the time and frequency domain comparison of the proposed 
controller with $H_{2} / H_{\infty}$ and PID controllers, the proposed controller shows better disturbance rejection properties while maintaining similar assistance properties.

Evaluation of the controller on the high-fidelity vehicle model accelerates the implementation of the controller for the hardware-in-the-loop (HIL) simulations. As a future work, the proposed controller will be implemented in a HIL simulation setup to verify the controller performance and robustness in a more realistic condition.

\section{ACKNOWLEDGMENTS}

The authors would like to thank the Ontario Centres of Excellence (OCE), the Natural Sciences and Engineering Research Council of Canada (NSERC), Toyota, and Maplesoft for their support of this research.

\section{References}

[1] Chen Z, Yang YL, Chen LH. Study on Boosting Curve for Electric Power Steering System Based on ADAMS. Advanced Materials Research. 2010;97 - 101:3308-3313.

[2] Zhou B, Bei SY, Zhao JB. Computer-Aided Design of Curved Assistance Characteristic of EPS System. Applied Mechanics and Materials. 2011;39:598-601.

[3] Zhao X, Shi X, Chen J, Li X. Dynamic Correction of the Steering-characteristic Curve and Application to an EPS Control System. International Journal of Automotive Technology. 2011;12(2):243-249.

[4] Lee MH, Ha SK, Choi JY, Yoon KS. Improvement of The Steering Feel of an Electric Power Steering System by Torque Map Modification. Journal of Mechanical Science and Technology. 2005;19(3):792-801.

[5] Xue-Ping Z, Xin L, Jie C, Jin-Lai M. Parametric Design and Application of Steering Characteristic Curve in Control for Electric Power Steering. Mechatronics. 2009;19(6):905-911.

[6] Mehrabi N, McPhee J. Steering Feel Improvement for Different Driver Types using Model-based Control. In: Proceedings of ASME 2014 International Design Engineering Technical Conferences and Computers and Information in Engineering Conference. Buffalo, New York; 2014. .

[7] Chabaan R, Wang L. Control of Electrical Power Assist Systems : $H_{\infty}$ Design, Torque Estimation and Structural Stability. JSAE Review. 2001;22:435-444.

[8] Daqing T, Guofu Y, Gang X. Model and $H_{\infty}$ Robust Control Design for Electric Power Steering System. In: Intelligent Mechatronics and Automation Conference. Chengdu, China; 2004. p. 779-783.

[9] Chabaan R. Optimal Control and Gain Scheduling of Electrical Power Steering Systems. IEEE Vehicle Power and Propulsion Conference. 2009 Sep;p. 53-59.

[10] Chabaan RC. $H_{\infty}$ Control Design and Gain Scheduling of Electrical Power Assist Steering Systems to Improve System Robustness and Stability. Wayne State University. Detroit, MI, USA; 2009.

[11] Parmar M, Hung JY. A Sensorless Optimal Control System for an Automotive Electric Power Assist Steering System. IEEE Transactions on Industrial Electronics. 2004 Apr;51(2):290-298.

[12] Peilin S, Shixiang G, Lidong M, Hongmin W, Shi P, Gao S, et al. Optimal Controller Design for Electric Power Steering System based on LQG. In: Information Engineering and Computer Science Conference. Wuhan, China; 2009. p. 1-4.

[13] Chitu C, Lackner J, Horn M, Pullagura PS, Waser H, Kohlböck M. Controller Design for an Electric Power Steering System based on LQR Techniques. COMPEL: The International Journal for Computation and Mathematics in Electrical and Electronic Engineering. 2013;32(3):763-775.

[14] Zhao W, Wang C. Mixed $H_{2} / H_{\infty}$ Road Feel Control of EPS based on Genetic Algorithm. SCIENCE CHINA Technological Sciences. 2012;55(1):72-80.

[15] El-shaer AH, Sugita S, Tomizuka M. Fixed-Structure Controller Design of Electric Power Steering Systems. In: American Control Conference. vol. 94720. St. Louis, Missouri; 2009. p. 445-450. 
[16] El-shaer AH, Tomizuka M. Robust Multi-Objective Control for Systems Involving Human-in-the-loop Passivity Constraints with Application to Electric Power Steering. In: 2010 IEEE/ASME International Conference on Advanced Intelligent Mechatronics. Montreal, Canada; 2010. p. 361-366.

[17] Tamura T, Maroonian A, Fuchs R. Active Compensation of Friction in Electric Power Steering. In: Proceedings of the FISITA 2012 World Automotive Congress. vol. 198. Springer Berlin Heidelberg; 2013. p. 213-225.

[18] Dannöhl C, Müller S, Ulbrich H. $H_{\infty}$-Control of a Rack-assisted Electric Power Steering System. Vehicle System Dynamics. 2012;50(4):527-544.

[19] Groll MV, Mueller S, Meister T, Tracht R. Disturbance Compensation with a Torque Controllable Steering System. Vehicle System Dynamics. 2006;44(4):327-338.

[20] Li L, He L, Du J, Lin T. An Electric Power Steering System Controller based on Disturbance Observer. In: IEEE International Conference on Integration Technology. Shenzhen, China; 2007. p. 446-449.

[21] Dong L, Kandula P, Gao Z, Wang D, Lili D, Zhiqiang G, et al. On a Robust Control System Design for an Electric Power Assist Steering System. In: American Control Conference. Baltimore, Maryland; 2010. p. 5356-5361.

[22] Mehrabi N, McPhee J, L Azad N. Optimal Disturbance Rejection Control Design for Electric Power Steering Systems. In: 50th IEEE Conference on Decision and Control and European Control Conference (CDC-ECC). Orlando, Florida; 2011. p. 6584-6589.

[23] Li X, Zhao XP, Chen J. Sliding Mode Control for Torque Ripple Reduction of an Electric Power Steering System based on a Reference Model. Proceedings of the Institution of Mechanical Engineers, Part D: Journal of Automobile Engineering. 2008;222(12):2283-2290.

[24] Knapczyk J, Maniowski M. Stiffness Synthesis of a Five-Rod Suspension for Given Load-Displacement Characteristics. Proceedings of the Institution of Mechanical Engineers, Part D: Journal of Automobile Engineering. 2006;220(7):879-889.

[25] Hall A, Uchida T, Loh F, Schmitke C, McPhee J. Reduction of a Vehicle Multibody Dynamic Model Using Homotopy Optimization. Archive of Mechanical Engineering. 2013;60(1):23-35.

[26] Hall A, McPhee J. Automation of Adams/Car K\&C Correlation using MATLAB. SAE Technical Paper 2014-01-0847. 2014;.

[27] Tamura T, Maroonian A, Higashi M, Fuchs R. Modeling and Simulation for Dynamic Analysis of Column Type Electric Power Steering. JTEKT Engineering journal. 2013;(1010E):19-25.

[28] Shin MC, Kim SH, Cho GH, Chu CN. Development of a Steering-system Model Considering Viscous Friction and its Verification. Proceedings of the Institution of Mechanical Engineers, Part D: Journal of Automobile Engineering. 2013;0(0):1-20.

[29] Armstrong B, Canudas DWC. Friction Modeling and Compensation. In: The Control Handbook. CRC Press; 1996. .

[30] Wong JY. Theory of Ground Vehicles. John Wiley \& Sons; 2001.

[31] ADAMS Online Help M. MSC Software, http://www.mscsoftware.com; 2011.

[32] Fiala E. Seitenkrafte am Rollenden Luftreifen. Zeitschrift, VDI. 1954;96(29):973-979.

[33] Kirk DE. Optimal Control Theory - an Introduction. Dover Publications; 2004.

[34] Lin CF. Advanced Control System Design. Prentice-Hall, Inc.; 1994.

[35] Sampson D, Cebon D. Active Roll Control of Single Unit Heavy Road Vehicles. Vehicle System Dynamics: International Journal of Vehicle Mechanics and Mobility. 2003;40(10):229-270.

[36] Zhao W, Shi G, Lin Y. Study on mixed $H_{\infty} / H_{2}$ control for enhancing the tracking performance of EPS. In: IEEE International Conference on Automation and Logistics. Jinan, China; 2007. p. 628-633.

[37] Chen XX, Yang T, Zhou K. A Generic Model-Based Advanced Control of Electric Power-Assisted Steering Systems. IEEE Transactions on Control Systems Technology. 2008;16(6):1289-1300.

[38] Saberi A, Sannuti P, Chen B. $H_{2}$ Optimal Control. Prentice-Hall, Inc.; 1995. 


\section{Appendix}

\section{A. State-space representation of the control-oriented model and the generalized system}

To construct the state-space representation of the control-oriented model, the state variables are defined as $\boldsymbol{x}=$ $\left[\begin{array}{lllllll}\beta & i & \omega_{z} & \theta_{p} & \dot{\theta}_{p} & \theta_{s w} & \dot{\theta}_{s w}\end{array}\right]^{T}$, and the inputs to the EPS system are defined as driver torque $\left(u_{\tau}\right)$, terminal voltage of electric motor $\left(u_{v}\right)$ and disturbance torque $\left(u_{d}\right)$. The state space equations of the system can be described by,

$$
\begin{gathered}
\dot{\boldsymbol{x}}=A \boldsymbol{x}+B_{v} u_{v}+B_{\tau} u_{\tau}+B_{d} u_{d} \\
\boldsymbol{y}=C \boldsymbol{x}
\end{gathered}
$$

where $A, B_{v}, B_{\tau}, B_{d}$ and $C$ are the system, input and output matrices of the system. Here, it is assumed that torque sensor value $\left(T_{t b}\right)$, steering wheel angle $\left(\theta_{s w}\right)$, motor current $(i)$, angular velocity of motor shaft $\left(\dot{\theta}_{m}\right)$, yaw rate $\left(\omega_{z}\right)$ and lateral acceleration $\left(a_{y}\right)$ of vehicle are measured and included in the output $\boldsymbol{y}$.

$$
B_{v}=\left[\begin{array}{c}
0 \\
L^{-1} \\
0 \\
0 \\
0 \\
0 \\
0
\end{array}\right], B_{\tau}=\left[\begin{array}{c}
0 \\
0 \\
0 \\
0 \\
0 \\
0 \\
J_{s w}^{-1}
\end{array}\right], B_{d}=\left[\begin{array}{c}
0 \\
0 \\
0 \\
0 \\
J_{p}^{-1} \\
0 \\
0
\end{array}\right]
$$

where the input matrices related to driver torque, disturbance torque, and terminal voltage are $B_{\tau}, B_{d}$, and $B_{v}$, respectively. The output matrix $\mathrm{C}$ can be described by:

$$
C=\left[\begin{array}{ccccccc}
0 & 0 & 0 & -K_{t b} & 0 & K_{t b} & 0 \\
0 & 0 & 0 & 0 & 0 & 1 & 0 \\
0 & 1 & 0 & 0 & 0 & 0 & 0 \\
0 & 0 & 0 & 0 & G & 0 & 0 \\
-\frac{\left(C_{\alpha f}+C_{\alpha r}\right)}{m} & 0 & -\frac{\left(C_{\alpha f} L_{f}-C_{\alpha r} L_{r}\right)}{m v_{x}} & \frac{C_{\alpha f}}{G_{\text {steering }} m} & 0 & 0 & 0 \\
0 & 0 & 1 & 0 & 0 & 0 & 0
\end{array}\right]
$$




$$
\begin{aligned}
& A=\left[\begin{array}{cccc}
-\frac{C_{\alpha f}+C_{\alpha r}}{m v_{x}} & 0 & -\left(m v_{x}+\frac{C_{\alpha f} L_{f}}{v_{x}}-\frac{C_{\alpha r} L_{r}}{v_{x}}\right) m^{-1} v_{x}{ }^{-1} & \frac{C_{\alpha f}}{G_{s t e e r i n g} m v_{x}} \\
0 & -\frac{R_{m}}{L_{m}} & 0 & 0 \\
-\frac{C_{\alpha f} L_{f}-C_{\alpha r} L_{r}}{I_{z z}} & 0 & -\left(\frac{L_{f}{ }^{2} C_{\alpha f}}{v_{x}}+\frac{L_{r}{ }^{2} C_{\alpha r}}{v_{x}}\right) I_{z z}{ }^{-1} & \frac{C_{\alpha f} L_{f}}{G_{s t e r i n g} I_{z z}} \\
0 & 0 & 0 & 0 \\
-\frac{C_{T \alpha}}{J_{p}} & \frac{K_{e} G}{J_{p}} & -\frac{C_{T \alpha} L_{f}}{v_{x} J_{p}} & -\left(K_{t b}+K_{p}-\frac{C_{T \alpha}}{G_{s t e r i n g}}\right) J_{p}{ }^{-1}
\end{array}\right. \\
& \begin{array}{lll}
0 & 0 & 0
\end{array} \\
& 0 \quad 0 \quad 0 \quad \frac{K_{t b}}{J_{s w}} \\
& \left.\begin{array}{ccc}
-\frac{K_{e} G}{L_{m}} & 0 & 0 \\
0 & 0 & 0 \\
1 & 0 & 0 \\
-\frac{b_{p}+b_{s w}+b_{t b}}{J_{p}} & \frac{K_{t b}}{J_{p}} & \frac{b_{t b}}{J_{p}} \\
0 & 0 & 1 \\
\frac{b_{t b}}{J_{s w}} & -\frac{K_{t b}}{J_{s w}} & -\frac{b_{s w}+b_{t b}}{J_{s w}}
\end{array}\right]
\end{aligned}
$$

and the controlled outputs $\left(z_{2}\right.$ and $\left.z_{\infty}\right)$ in the $H_{2} / H_{\infty}$ controller are defined as follows:

$$
\left\{\begin{array}{c}
z_{\infty}=C_{1} \boldsymbol{x}+D_{11} \boldsymbol{\omega}+D_{12} u_{v} \\
z_{2}=C_{2} \boldsymbol{x}+D_{21} \boldsymbol{\omega}+D_{22} u_{v}
\end{array}\right.
$$

where the system matrices without the weight functions are described by:

$$
\begin{gathered}
C_{1}=\left[\begin{array}{ccccccc}
0 & 0 & 0 & -K_{t b} & 0 & K_{t b} & 0
\end{array}\right] \\
C_{2}=\left[\begin{array}{ccccccc}
0 & G K_{e} & 0 & 0 & 0 & 0 & 0 \\
0 & 0 & 0 & 0 & 0 & 0 & 0
\end{array}\right] \\
D_{11}=\left[\begin{array}{ll}
0 & 0
\end{array}\right], D_{12}=0 \\
D_{21}=\left[\begin{array}{cc}
-K_{a} & 0 \\
0 & 0
\end{array}\right], D_{22}=\left[\begin{array}{l}
0 \\
1
\end{array}\right]
\end{gathered}
$$




\section{B. Steering System Model Parameters}

The model parameters used in the control-oriented model and the high-fidelity model can be found in Table 1 .

Table 1: List of parameters used in the high-fidelity and control-oriented models

\begin{tabular}{|c|c|c|c|}
\hline Parameters & Description & Value & Unit \\
\hline$K_{t b}$ & torsion bar stiffness & 117 & {$[\mathrm{~N} . \mathrm{m} / \mathrm{rad}]$} \\
\hline$b_{t b}$ & torsion bar damping & 2.2 & [N.m.s/rad] \\
\hline$\theta_{1}$ & lower steering shaft angle & 118.6 & [degree] \\
\hline$\gamma$ & universal joint bending angle & 22.3 & [degree] \\
\hline$L_{m}$ & electric motor inductance coefficient & $1.510^{-5}$ & {$[\mathrm{H}]$} \\
\hline$R_{m}$ & electric motor resistance coefficient & 0.15 & [ohms] \\
\hline$K_{e}$ & electric motor back electromotive force $(\mathrm{emf})$ coefficient & 0.02 & [V.s/rad] \\
\hline$n, v_{0}, v_{s}$ & shaping factors in the friction force & $1,10^{-4}, 10^{-3}$ & {$[-]$} \\
\hline$b_{s w}, a_{s w}$ & viscous friction coefficients of the steering wheel & $0.008,1$ & [N.m.s/rad, - ] \\
\hline$T_{c, s w}, T_{s, s w}$ & coulomb friction coefficients of the steering wheel & $0.0363,0.05$ & {$[\mathrm{~N} . \mathrm{m}]$} \\
\hline$b_{r}, a_{r}$ & viscous friction coefficients of the rack & 78,1 & [N.s/m, - ] \\
\hline$F_{c, r}, F_{s, r}$ & coulomb friction coefficients of the rack & $141.9,130$ & {$[\mathrm{~N}]$} \\
\hline$J_{s w}$ & inertia of steering wheel & 0.0009 & {$\left[\mathrm{~kg} \cdot \mathrm{m}^{2}\right]$} \\
\hline$J_{p}$ & inertia of steering system at pinion & 0.01258 & {$\left[\mathrm{~kg} \cdot \mathrm{m}^{2}\right]$} \\
\hline$b_{p}$ & viscous damping of the pinion & 3.715 & [N.m.s/rad] \\
\hline$K_{p}$ & stiffness induced by inclined kingpin axis & 71.4 & {$[\mathrm{~N} . \mathrm{m} / \mathrm{rad}]$} \\
\hline$G$ & worm gear ratio & 20 & {$[-]$} \\
\hline$G_{\text {steering }}$ & average steering ratio & 15.29 & {$[-]$} \\
\hline$L_{f}$ & the distance from front tire to vehicle's center of mass & 1.192 & [m] \\
\hline$L_{r}$ & the distance from rear tire to vehicle's center of mass & 1.548 & {$[\mathrm{~m}]$} \\
\hline$m$ & vehicle mass & 2077 & {$[\mathrm{~kg}]$} \\
\hline$I_{z z}$ & vehicle yaw inertia & 1995.78 & {$\left[\mathrm{~kg} \cdot \mathrm{m}^{2}\right]$} \\
\hline$C_{T \alpha}$ & self-aligning-torque stiffness & 2000 & [N.m/rad] \\
\hline$C_{\alpha[f, r]}$ & front and rear tire cornering stiffness & 117000 & {$[\mathrm{~N} / \mathrm{rad}]$} \\
\hline
\end{tabular}

